\title{
Hermite-Hadamard integral inequalities on coordinated convex functions in quantum calculus
}

\author{
Manar A. Alqudah ${ }^{1}$ (D), Artion Kashuri² (D), Pshtiwan Othman Mohammed ${ }^{3 *} \mathbb{D}$, Thabet Abdeljawad Th, $^{4,6^{*}}$ (D), \\ Muhammad Raees ${ }^{7}$, Matloob Anwar ${ }^{7}$ and Y.S. Hamed ${ }^{8}$ (D)
}

"Correspondence:

pshtiwansangawi@gmail.com; tabdeljawad@psu.edu.sa

${ }^{3}$ Department of Mathematics, College of Education, University of Sulaimani, Sulaimani, Kurdistan Region, Iraq

${ }^{4}$ Department of Mathematics and General Sciences, Prince Sultan University, P.O. Box 66833, Riyadh 11586, Saudi Arabia Full list of author information is available at the end of the article

\section{Springer}

\begin{abstract}
At first, we recall the $q$-operators in the context of $q$-calculus and by examining these operators we will introduce new definitions of the partial $q$-operators. Then, we investigate some new refinements inequalities of Hermite-Hadamard $(H-H)$ type on the coordinated convex functions involving the new defined partial $q$-operators. From our main results, we establish several specific inequalities and we point out the existing results which had already been obtained in the literature.
\end{abstract}

MSC: 26A51; 26A33; 26D07; 26D10; 26D15

Keywords: Hermite-Hadamard inequality; Coordinated convex functions; Quantum calculus

\section{Introduction and Preliminaries}

Let $\Psi$ be defined on an interval $J \subseteq \mathbb{R}_{e}\left(\mathbb{R}_{e}\right.$ is the set of real numbers), then $\Psi$ is convex if

$$
\Psi(\lambda u+(1-\lambda) w) \leq \lambda \Psi(u)+(1-\lambda) \Psi(w)
$$

for all $u, w$ in $J$ and for any $\lambda$ in $[0,1]$.

Convex functions plays a vital role in the development of many fields of mathematics and significant applications are found in a variety of applied sciences such as optimization theory, number theory, combinatorics, special means theory, approximation theory and numerical analysis, see [1-5]. The well-known Hermite-Hadamard $(H-H)$ inequality has central part in this development as it gives the criterion for convex functions. The $H-H$ inequality is given as follows: Let $\Psi:[A, B] \subset \mathbb{R}_{e} \rightarrow \mathbb{R}_{e}$ be a convex function with $A<B$, then the $H-H$ inequality is given as [6]

$$
\Psi\left(\frac{A+B}{2}\right) \leq \frac{1}{B-A} \int_{A}^{B} \Psi(\lambda) d \lambda \leq \frac{\Psi(A)+\Psi(B)}{2} .
$$

The $H-H$ inequality (1.2) is converted into three equivalent integral inequalities by the help of Riemann-Liouville fractional operators; see [7-9]. Also, it has been generalized

(c) The Author(s) 2021. This article is licensed under a Creative Commons Attribution 4.0 International License, which permits use, sharing, adaptation, distribution and reproduction in any medium or format, as long as you give appropriate credit to the original author(s) and the source, provide a link to the Creative Commons licence, and indicate if changes were made. The images or other third party material in this article are included in the article's Creative Commons licence, unless indicated otherwise in a credit line to the material. If material is not included in the article's Creative Commons licence and your intended use is not permitted by statutory regulation or exceeds the permitted use, you will need to obtain permission directly from the copyright holder. To view a copy of this licence, visit http://creativecommons.org/licenses/by/4.0/ 
and converted into many other integral inequalities by the help of other types of fractional operators, see [10-13].

In 2001, Dragomir [14] introduced the notion of coordinated convex functions in a rectangle from of the plane $\mathbb{R}_{e}^{2}$.

Definition 1.1 ([14]) Let $\Delta:=[A, B] \times[C, D]$ be a bi-dimensional interval such that $0 \leq$ $A<B<\infty, 0 \leq C<D<\infty$. Then, a function $\Psi: \Delta \rightarrow \mathbb{R}_{e}$ is called coordinated convex on $\Delta$, if the partial mappings $\Psi_{v}:[A, B] \rightarrow \mathbb{R}_{e}, \Psi_{v}(x)=\Psi(x, v)$ and $\Psi_{u}:[C, D] \rightarrow \mathbb{R}_{e}, \Psi_{u}(y)=$ $\Psi(u, y)$ are convex for each $y, v \in[C, D]$ and $x, u \in[A, B]$. Also, $\Psi$ satisfies the inequality

$$
\Psi(\lambda u+(1-\lambda) w, \lambda v+(1-\lambda) z) \leq \lambda \Psi(u, v)+(1-\lambda) \Psi(w, z)
$$

for all $(u, v),(w, z) \in \Delta$ and $\lambda \in[0,1]$.

By the help of above definition, Dragomir [14] established the following $H-H$ type inequalities similar to the one dimensional case.

Theorem 1.1 ([14]) Suppose that $\Psi: \Delta \rightarrow \mathbb{R}_{e}$ is a coordinated convex function on $\Delta$. Then we have

$$
\begin{aligned}
& \Psi\left(\frac{A+B}{2}, \frac{C+D}{2}\right) \\
& \quad \leq \frac{1}{2}\left[\frac{1}{B-A} \int_{A}^{B} \Psi\left(u, \frac{C+D}{2}\right) d u+\frac{1}{D-C} \int_{C}^{D} \Psi\left(\frac{A+B}{2}, v\right) d v\right] \\
& \quad \leq \frac{1}{(B-A)(D-C)} \int_{A}^{B} \int_{C}^{D} \Psi(u, v) d u d v \\
& \quad \leq \frac{1}{2}\left[\frac{1}{B-A} \int_{A}^{B}[\Psi(u, C)+\Psi(u, D)] d u+\frac{1}{D-C} \int_{C}^{D}[\Psi(A, v)+\Psi(B, v)] d v\right] \\
& \quad \leq \frac{\Psi(A, C)+\Psi(A, D)+\Psi(B, C)+\Psi(B, D)}{4} .
\end{aligned}
$$

Many relevant results have been reported in this direction with different class of convex functions; see [15-19] and the references therein.

In the early 20th century, Jackson $[20,21]$ worked on the classical notion of a derivative without limit allowing for easier study of number theory and ordinary calculus in his investigations. Jackson got the credit of the $q$-analogue of the various well-known results of calculus; see [20-24].

For a real valued function $\Psi$, the $q$-derivative is characterized by

$$
\mathbf{D}_{q} \Psi(\varsigma)=\frac{\Psi(q \varsigma)-\Psi(\varsigma)}{q \varsigma-\varsigma}
$$

where $q \in(0,1)$. The well-known Jackson integral of a real valued function $\Psi$ is given by the following series expansion:

$$
\int_{0}^{\mu} \Psi(\varsigma) d_{q} \varsigma=(1-q) \mu \sum_{r=0}^{\infty} q^{r} \Psi\left(q^{r} \mu\right)
$$


References $[25,26]$ discuss the notion of $q$-derivatives and $q$-integrals over the finite interval $\left[k_{1}, k_{2}\right]$ of real numbers and defined the $q_{k_{1}}$-derivative and $q_{k_{1}}$-integral.

Definition $1.2([25,26])$ For any continuous function $\Psi:\left[k_{1}, k_{2}\right] \rightarrow \mathbb{R}_{e}$ and $q \in(0,1)$, the $q_{k_{1}}$-derivative of $\Psi$ at $\varsigma \in\left[k_{1}, k_{2}\right]$ is defined by

$$
{ }_{k_{1}} \mathbf{D}_{q} \Psi(\varsigma)=\frac{\Psi(\varsigma)-\Psi\left(q \varsigma+(1-q) k_{1}\right)}{(1-q)\left(\varsigma-k_{1}\right)}, \quad \varsigma \neq k_{1} .
$$

Definition $1.3([25,26])$ For a continuous function $\Psi:\left[k_{1}, k_{2}\right] \rightarrow \mathbb{R}_{e}$ and $q \in(0,1)$, the $q_{k_{1}}$-integral of $\Psi$ at $\varsigma \in\left[k_{1}, k_{2}\right]$ is defined by

$$
\int_{k_{1}}^{k} \Psi(\varsigma)_{k_{1}} d_{q} \varsigma=(1-q)\left(k-k_{1}\right) \sum_{h=0}^{\infty} q^{h} \Psi\left(q^{h} k+\left(1-q^{h}\right) k_{1}\right), \quad k \in\left[k_{1}, k_{2}\right] .
$$

If $k_{1}=0$, then

$$
\int_{0}^{k} \Psi(\varsigma)_{0} d_{q} \varsigma=(1-q) k \sum_{h=0}^{\infty} q^{h} \Psi\left(q^{h} k\right)=\int_{0}^{k} \Psi(\varsigma) d_{q} \varsigma
$$

which is the classical $q$-integral (1.6).

In view of the above definitions, the authors [27] established the following inequality.

Theorem 1.2 ([27]) Let $\Psi:[A, B] \rightarrow \mathbb{R}_{e}$ be a convex differentiable function on $[A, B]$ and $q \in(0,1)$. Then we have

$$
\Psi\left(\frac{q A+B}{1+q}\right) \leq \frac{1}{B-A} \int_{A}^{B} \Psi(\varsigma)_{A} d_{q} \varsigma \leq \frac{q \Psi(A)+\Psi(B)}{1+q} .
$$

In [28], the authors generalize the notion of $q$-derivatives and $q$-integrals by introducing the $q^{k_{2}}$-derivative and $q^{k_{2}}$-integral over the finite real interval $\left[k_{1}, k_{2}\right]$.

Definition 1.4 ([28]) For any continuous function $\Psi:\left[k_{1}, k_{2}\right] \rightarrow \mathbb{R}_{e}$ and $q \in(0,1)$, the $q^{k_{2}}$-derivative of $\Psi$ at $\varsigma \in\left[k_{1}, k_{2}\right]$ is defined by

$$
{ }^{k_{2}} \mathbf{D}_{q} \Psi(\varsigma)=\frac{\Psi(\varsigma)-\Psi\left(q \varsigma+(1-q) k_{2}\right)}{(1-q)\left(\varsigma-k_{2}\right)}, \quad \varsigma \neq k_{2} .
$$

Definition 1.5 ([28]) For any continuous function $\Psi:\left[k_{1}, k_{2}\right] \rightarrow \mathbb{R}_{e}$ and $q \in(0,1)$, the $q^{k_{2}}$-integral of $\Psi$ at $\varsigma \in\left[k_{1}, k_{2}\right]$ is defined by

$$
\int_{k}^{k_{2}} \Psi(\varsigma)^{k_{2}} d_{q} \varsigma=(1-q)\left(k_{2}-k\right) \sum_{h=0}^{\infty} q^{h} \Psi\left(q^{h} k+\left(1-q^{h}\right) k_{2}\right), \quad k \in\left[k_{1}, k_{2}\right] .
$$

In [28], the authors established the following counterpart for integrals (1.10). 
Theorem 1.3 ([28]) Let $\Psi:[A, B] \rightarrow \mathbb{R}_{e}$ be a convex differentiable function on $[A, B]$ and $q \in(0,1)$. Then we have

$$
\Psi\left(\frac{A+q B}{1+q}\right) \leq \frac{1}{B-A} \int_{A}^{B} \Psi(\varsigma)^{B} d_{q} \varsigma \leq \frac{\Psi(A)+q \Psi(B)}{1+q} .
$$

In [29], the authors extended the definition of $q$-derivative and $q$-integral to the case of two variables.

Definition 1.6 ([29]) Let $\Psi: \Delta \rightarrow \mathbb{R}_{e}$ be a continuous function of two variables and $0<q_{1}<1,0<q_{2}<1$. Then, the partial $q_{1 A}$-derivative, partial $q_{2 C}$-derivative and partial $q_{1 A} q_{2 C}$ - derivatives are defined by

$$
\begin{array}{ll}
\frac{{ }_{A} \partial_{q_{1}} \Psi(u, v)}{{ }_{A} \partial_{q_{1}} u}=\frac{\Psi(u, v)-\Psi\left(q_{1} u+\left(1-q_{1}\right) A, v\right)}{\left(1-q_{1}\right)(u-A)}, & u \neq A, \\
\frac{{ }_{C} \partial_{q_{2}} \Psi(u, v)}{{ }_{C} \partial_{q_{2}} u}=\frac{\Psi(u, v)-\Psi\left(u, q_{2} v+\left(1-q_{2}\right) C\right)}{\left(1-q_{2}\right)(v-C)}, & v \neq C,
\end{array}
$$

and

$$
\begin{aligned}
\frac{A, C}{{ }_{A} \partial_{q_{1} q_{2}} \Psi(u, v)}{ }_{A} \partial_{q_{1}} u_{C} \partial_{q_{2}} v & \frac{1}{\left(1-q_{1}\right)(u-A)\left(1-q_{2}\right)(v-C)}\left[\Psi\left(q_{1} u+\left(1-q_{1}\right) A, q_{2} v+\left(1-q_{2}\right) C\right)\right. \\
& -\Psi\left(q_{1} u+\left(1-q_{1}\right) A, v\right)-\Psi\left(u, q_{2} v+\left(1-q_{2}\right) C\right) \\
& +\Psi(u, v)], \quad u \neq A, v \neq C .
\end{aligned}
$$

Definition 1.7 ([29]) Let $\Psi: \Delta \rightarrow \mathbb{R}_{e}$ be a continuous function of two variables and $0<$ $q_{1}<1,0<q_{2}<1$, Then the definite $q_{1 A} q_{2 C}$-integral on $\Delta$ is defined by

$$
\begin{aligned}
\int_{A}^{v} & \int_{C}^{\mu} \Psi(u, v)_{C} d_{q_{2}} v_{A} d_{q_{1}} u \\
= & \left(1-q_{1}\right)\left(1-q_{2}\right)(v-A)(\mu-C) \\
& \quad \times \sum_{h=0}^{\infty} \sum_{k=0}^{\infty} q_{1}^{k} q_{2}^{h} \Psi\left(q_{1}^{k} v+\left(1-q_{1}^{k}\right) A, q_{2}^{h} \mu+\left(1-q_{2}^{h}\right) C\right),
\end{aligned}
$$

for all $(\nu, \mu) \in \Delta$.

Meanwhile, the authors [29] proved the following inequality.

Theorem 1.4 ([29]) Let $\Psi: \Delta \rightarrow \mathbb{R}_{e}$ be a coordinated convex function on $\Delta$, then the following inequalities hold:

$$
\begin{aligned}
& \Psi\left(\frac{A+B}{2}, \frac{C+D}{2}\right) \\
& \quad \leq \frac{1}{2}\left[\frac{1}{B-A} \int_{A}^{B} \Psi\left(u, \frac{C+D}{2}\right){ }_{A} d_{q_{1}} u+\frac{1}{D-C} \int_{C}^{D} \Psi\left(\frac{A+B}{2}, v\right)_{C} d_{q_{2}} v\right] \\
& \quad \leq \frac{1}{(B-A)(D-C)} \int_{A}^{B} \int_{C}^{D} \Psi(u, v)_{C} d_{q_{2}} v_{A} d_{q_{1}} u
\end{aligned}
$$




$$
\begin{aligned}
\leq & \frac{q_{2}}{2\left(1+q_{2}\right)(B-A)} \int_{A}^{B} \Psi(u, C)_{A} d_{q_{1}} u \\
& +\frac{1}{2\left(1+q_{2}\right)(B-A)} \int_{A}^{B} \Psi(u, D)_{A} d_{q_{1}} u+\frac{q_{1}}{2\left(1+q_{1}\right)(D-C)} \int_{C}^{D} \Psi(A, v)_{C} d_{q_{2}} v \\
& +\frac{1}{2\left(1+q_{1}\right)(D-C)} \int_{C}^{D} \Psi(B, v)_{C} d_{q_{2}} v \\
\leq & \frac{q_{1} q_{2} \Psi(A, C)+q_{1} \Psi(A, D)+q_{2} \Psi(B, C)+\Psi(B, D)}{\left(1+q_{1}\right)\left(1+q_{2}\right)}
\end{aligned}
$$

Recently, in [30], the authors disproved the inequality (1.18) by giving a counter example and proved the following correct $H-H$ inequality.

Theorem 1.5 ([30]) Let $\Psi: \Delta \rightarrow \mathbb{R}_{e}$ be a coordinated convex function on $\Delta$, then for all $q_{1}, q_{2} \in(0,1)$, we have

$$
\begin{aligned}
\Psi( & \left.\frac{q_{1} A+B}{1+q_{1}}, \frac{q_{2} C+D}{1+q_{2}}\right) \\
\leq & \frac{1}{2}\left[\frac{1}{B-A} \int_{A}^{B} \Psi\left(u, \frac{q_{2} C+D}{1+q_{2}}\right){ }_{A} d_{q_{1}} u+\frac{1}{D-C} \int_{C}^{D} \Psi\left(\frac{q_{1} A+B}{1+q_{1}}, v\right){ }_{C} d_{q_{2}} v\right] \\
\leq & \frac{1}{(B-A)(D-C)} \int_{A}^{B} \int_{C}^{D} \Psi(u, v)_{C} d_{q_{2}} v_{A} d_{q_{1}} u \\
\leq & \frac{q_{2}}{2\left(1+q_{2}\right)(B-A)} \int_{A}^{B} \Psi(u, C)_{A} d_{q_{1}} u \\
& +\frac{1}{2\left(1+q_{2}\right)(B-A)} \int_{A}^{B} \Psi(u, D)_{A} d_{q_{1}} u+\frac{q_{1}}{2\left(1+q_{1}\right)(D-C)} \int_{C}^{D} \Psi(A, v)_{C} d_{q_{2}} v \\
& +\frac{1}{2\left(1+q_{1}\right)(D-C)} \int_{C}^{D} \Psi(B, v)_{C} d_{q_{2}} v \\
\leq & \frac{q_{1} q_{2} \Psi(A, C)+q_{1} \Psi(A, D)+q_{2} \Psi(B, C)+\Psi(B, D)}{\left(1+q_{1}\right)\left(1+q_{2}\right)} .
\end{aligned}
$$

Now, by combining the two concepts of $q$-derivatives and $q$-integrals given in Definitions 1.2-1.7, we present the following mixed types of partial derivatives and integrals in the two variables along with some examples.

Definition 1.8 Let $\Psi: \Delta \rightarrow \mathbb{R}_{e}$ be a continuous function of two variables and $0<q_{1}<1$, $0<q_{2}<1$, Then the partial $q_{1}^{B}$-derivative, partial $q_{2}^{D}$-derivative and partial $q_{1}^{B} q_{2}^{D}, q_{1}^{B} q_{2 C}$ and $q_{1 A} q_{2}^{D}$ derivatives are defined, respectively, by

$$
\begin{aligned}
& \frac{{ }^{B} \partial_{q_{1}} \Psi(u, v)}{{ }^{B} \partial_{q_{1}} u}=\frac{\Psi(u, v)-\Psi\left(q_{1} u+\left(1-q_{1}\right) B, v\right)}{\left(1-q_{1}\right)(u-B)}, \quad u \neq B, \\
& \frac{D \partial_{q_{2}} \Psi(u, v)}{D \partial_{q_{2}} u}=\frac{\Psi(u, v)-\Psi\left(u, q_{2} v+\left(1-q_{2}\right) D\right)}{\left(1-q_{2}\right)(v-D)}, \quad v \neq D, \\
& \frac{B, D \partial_{q_{1} q_{2}} \Psi(u, v)}{B \partial_{q_{1}} u^{D} \partial_{q_{2}} v} \\
& =\frac{1}{\left(1-q_{1}\right)(u-B)\left(1-q_{2}\right)(v-D)}\left[\Psi\left(q_{1} u+\left(1-q_{1}\right) B, q_{2} v+\left(1-q_{2}\right) D\right)\right.
\end{aligned}
$$




$$
\begin{aligned}
& -\Psi\left(q_{1} u+\left(1-q_{1}\right) B, v\right)-\Psi\left(u, q_{2} v+\left(1-q_{2}\right) D\right) \\
& +\Psi(u, v)], \quad u \neq B, v \neq D, \\
& \frac{{ }_{C}^{B} \partial_{q_{1} q_{2}} \Psi(u, v)}{{ }^{B} \partial_{q_{1}} u_{C} \partial_{q_{2}} v} \\
& =\frac{1}{\left(1-q_{1}\right)(u-B)\left(1-q_{2}\right)(v-C)}\left[\Psi\left(q_{1} u+\left(1-q_{1}\right) B, q_{2} v+\left(1-q_{2}\right) C\right)\right. \\
& -\Psi\left(q_{1} u+\left(1-q_{1}\right) B, v\right)-\Psi\left(u, q_{2} v+\left(1-q_{2}\right) C\right) \\
& +\Psi(u, v)], \quad u \neq B, v \neq C,
\end{aligned}
$$

and

$$
\begin{aligned}
& \frac{{ }_{A}^{D} \partial_{q_{1} q_{2}} \Psi(u, v)}{{ }_{A} \partial_{q_{1}} u^{D} \partial_{q_{2}} v} \\
& =\frac{1}{\left(1-q_{1}\right)(u-A)\left(1-q_{2}\right)(v-D)}\left[\Psi\left(q_{1} u+\left(1-q_{1}\right) A, q_{2} v+\left(1-q_{2}\right) D\right)\right. \\
& \quad-\Psi\left(q_{1} u+\left(1-q_{1}\right) A, v\right)-\Psi\left(u, q_{2} v+\left(1-q_{2}\right) D\right) \\
& \quad+\Psi(u, v)], \quad u \neq A, v \neq D .
\end{aligned}
$$

Definition 1.9 Let $\Psi: \Delta \rightarrow \mathbb{R}_{e}$ be a continuous function of two variables and $0<q_{1}<1$, $0<q_{2}<1$, Then the definite $q_{1}^{B} q_{2}^{D}$-integral, $q_{1 A} q_{2}^{D}$-integral and $q_{1}^{B} q_{2 C}$-integral on $\Delta$ are defined by

$$
\begin{aligned}
& \int_{v}^{B} \int_{\mu}^{D} \Psi(u, v)^{D} d_{q_{2}} v^{B} d_{q_{1}} u \\
& =\left(1-q_{1}\right)\left(1-q_{2}\right)(B-v)(D-\mu) \\
& \quad \times \sum_{h=0}^{\infty} \sum_{k=0}^{\infty} q_{1}^{k} q_{2}^{h} \Psi\left(q_{1}^{k} v+\left(1-q_{1}^{k}\right) B, q_{2}^{h} \mu+\left(1-q_{2}^{h}\right) D\right), \quad \forall(v, \mu) \in \Delta, \\
& \int_{A}^{v} \int_{\mu}^{D} \Psi(u, v)^{D} d_{q_{2}} v_{A} d_{q_{1}} u \\
& =\left(1-q_{1}\right)\left(1-q_{2}\right)(v-A)(D-\mu) \\
& \quad \times \sum_{h=0}^{\infty} \sum_{k=0}^{\infty} q_{1}^{k} q_{2}^{h} \Psi\left(q_{1}^{k} v+\left(1-q_{1}^{k}\right) A, q_{2}^{h} \mu+\left(1-q_{2}^{h}\right) D\right), \quad \forall(v, \mu) \in \Delta,
\end{aligned}
$$

and

$$
\begin{aligned}
\int_{v}^{B} \int_{C}^{\mu} \Psi(u, v)_{C} d_{q_{2}} v^{B} d_{q_{1}} u \\
=\left(1-q_{1}\right)\left(1-q_{2}\right)(B-v)(\mu-C) \\
\quad \times \sum_{h=0}^{\infty} \sum_{k=0}^{\infty} q_{1}^{k} q_{2}^{h} \Psi\left(q_{1}^{k} v+\left(1-q_{1}^{k}\right) B, q_{2}^{h} \mu+\left(1-q_{2}^{h}\right) C\right), \quad \forall(v, \mu) \in \Delta .
\end{aligned}
$$


Example 1.1 All the $q_{1} q_{2}$-integrals are different for general functions. For instance,

$$
\begin{aligned}
& \int_{A}^{B} \int_{C}^{D} u v^{D} d_{q_{2}} v^{B} d_{q_{1}} u=\frac{(B-A)\left(A+q_{1} B\right)(D-C)\left(C+q_{2} D\right)}{\left(1+q_{1}\right)\left(1+q_{2}\right)}, \\
& \int_{A}^{B} \int_{C}^{D} u v^{D} d_{q_{2}} v_{A} d_{q_{1}} u=\frac{(B-A)\left(q_{1} A+B\right)(D-C)\left(C+q_{2} D\right)}{\left(1+q_{1}\right)\left(1+q_{2}\right)}, \\
& \int_{A}^{B} \int_{C}^{D} u v_{C} d_{q_{2}} v^{B} d_{q_{1}} u=\frac{(B-A)\left(A+q_{1} B\right)(D-C)\left(q_{2} C+D\right)}{\left(1+q_{1}\right)\left(1+q_{2}\right)}, \\
& \int_{A}^{B} \int_{C}^{D} u v_{C} d_{q_{2}} v_{A} d_{q_{1}} u=\frac{(B-A)\left(q_{1} A+B\right)(D-C)\left(q_{2} C+D\right)}{\left(1+q_{1}\right)\left(1+q_{2}\right)} .
\end{aligned}
$$

Furthermore,

$$
\int_{C}^{B} \int_{C}^{D} u v d v d u=\frac{\left(B^{2}-A^{2}\right)\left(D^{2}-C^{2}\right)}{4},
$$

subject to the condition that both $q_{1}, q_{2} \rightarrow 1^{-}$.

Now, we obtain midpoint type inequalities from the inequality (1.19).

Remark 1.1 We have four special cases for this inequality at midpoint.

- If $A=a, B=\frac{q_{1} a+b}{1+q_{1}}, C=c, D=\frac{q_{2} d+c}{1+q_{2}}$, then

$$
\begin{aligned}
\Psi( & \left.\frac{\left(2 q_{1}+q_{1}^{2}\right) a+b}{\left(1+q_{1}\right)^{2}}, \frac{\left(1+q_{2}+q_{2}^{2}\right) c+q_{2} d}{\left(1+q_{2}\right)^{2}}\right) \\
\leq & \frac{1+q_{1}}{2(b-a)} \int_{a}^{\frac{q_{1} a+b}{1+q_{1}}} \Psi\left(u, \frac{\left(1+q_{2}+q_{2}^{2}\right) c+q_{2} d}{\left(1+q_{2}\right)^{2}}\right){ }_{a} d_{q_{1}} u \\
& +\frac{1+q_{2}}{2 q_{2}(d-c)} \int_{c}^{\frac{q_{2} d+c}{1+q_{2}}} \Psi\left(\frac{\left(2 q_{1}+q_{1}^{2}\right) a+b}{\left(1+q_{1}\right)^{2}}, v\right){ }_{c} d_{q_{2}} v \\
\leq & \frac{\left(1+q_{1}\right)\left(1+q_{2}\right)}{q_{2}(b-a)(d-c)} \int_{a}^{\frac{q_{1} a+b}{1+q_{1}}} \int_{c}^{\frac{q_{2} d+c}{1+q_{2}}} \Psi(u, v)_{c} d_{q_{2}} v_{a} d_{q_{1}} u \\
\leq & \frac{q_{2}\left(1+q_{1}\right)}{2\left(1+q_{2}\right)(b-a)} \int_{a}^{\frac{q_{1} a+b}{1+q_{1}}} \Psi(u, c)_{a} d_{q_{1}} u \\
& +\frac{1+q_{1}}{2\left(1+q_{2}\right)(b-a)} \int_{a}^{\frac{q_{1} a+b}{1+q_{1}}} \Psi\left(u, \frac{q_{2} d+c}{1+q_{2}}\right){ }_{a} d_{q_{1}} u \\
& +\frac{q_{1}\left(1+q_{2}\right)}{2 q_{2}\left(1+q_{1}\right)(d-c)} \int_{c}^{\frac{q_{2} d+c}{1+q_{2}}} \Psi(a, v)_{c} d_{q_{2}} v \\
& +\frac{1+q_{2}}{2 q_{2}\left(1+q_{1}\right)(d-c)} \int_{c}^{\frac{q_{2} d+c}{1+q_{2}}} \Psi\left(\frac{q_{1} a+b}{1+q_{1}}, v\right){ }_{c} d_{q_{2}} v \\
\leq & \frac{q_{1} q_{2} \Psi(a, c)+q_{1} \Psi\left(a, \frac{q_{2} d+c}{1+q_{2}}\right)+q_{2} \Psi\left(\frac{q_{1} a+b}{1+q_{1}}, c\right)+\Psi\left(\frac{q_{1} a+b}{1+q_{1}}, \frac{q_{2} d+c}{1+q_{2}}\right)}{\left(1+q_{1}\right)\left(1+q_{2}\right)} .
\end{aligned}
$$


- If $A=a, B=\frac{q_{1} b+a}{1+q_{1}}, C=c, D=\frac{q_{2} d+c}{1+q_{2}}$, then

$$
\begin{aligned}
& \Psi\left(\frac{\left(1+q_{1}+q_{1}^{2}\right) a+q_{1} b}{\left(1+q_{1}\right)^{2}}, \frac{\left(1+q_{2}+q_{2}^{2}\right) c+q_{2} d}{\left(1+q_{2}\right)^{2}}\right) \\
& \leq \frac{1+q_{1}}{2 q_{1}(b-a)} \int_{a}^{\frac{q_{1} b+a}{1+q_{1}}} \Psi\left(u, \frac{\left(1+q_{2}+q_{2}^{2}\right) c+q_{2} d}{\left(1+q_{2}\right)^{2}}\right){ }_{a} d_{q_{1}} u \\
& +\frac{1+q_{2}}{2 q_{2}(d-c)} \int_{c}^{\frac{q_{2} d+c}{1+q_{2}}} \Psi\left(\frac{\left(1+q_{1}+q_{1}^{2}\right) a+q_{1} b}{\left(1+q_{1}\right)^{2}}, v\right){ }_{c} d_{q_{2}} v \\
& \leq \frac{\left(1+q_{1}\right)\left(1+q_{2}\right)}{q_{1} q_{2}(b-a)(d-c)} \int_{a}^{\frac{q_{1} b+a}{1+q_{1}}} \int_{c}^{\frac{q_{2} d+c}{1+q_{2}}} f(u, v){ }_{c} d_{q_{2}} v_{a} d_{q_{1}} u \\
& \leq \frac{q_{2}\left(1+q_{1}\right)}{2 q_{1}\left(1+q_{2}\right)(b-a)} \int_{a}^{\frac{q_{1} b+a}{1+q_{1}}} \Psi(u, c){ }_{a} d_{q_{1}} u \\
& +\frac{1+q_{1}}{2 q_{1}\left(1+q_{2}\right)(b-a)} \int_{a}^{\frac{q_{1} b+a}{1+q_{1}}} \Psi\left(u, \frac{q_{2} d+c}{1+q_{2}}\right){ }_{a} d_{q_{1}} u \\
& +\frac{q_{1}\left(1+q_{2}\right)}{2 q_{2}\left(1+q_{1}\right)(d-c)} \int_{c}^{\frac{q_{2} d+c}{1+q_{2}}} \Psi(a, v){ }_{c} d_{q_{2}} v \\
& +\frac{1+q_{2}}{2 q_{2}\left(1+q_{1}\right)(d-c)} \int_{c}^{\frac{q_{2} d+c}{1+q_{2}}} \Psi\left(\frac{q_{1} a+b}{1+q_{1}}, v\right){ }_{c} d_{q_{2}} v \\
& \leq \frac{q_{1} q_{2} \Psi(a, c)+q_{1} \Psi\left(a, \frac{q_{2} d+c}{1+q_{2}}\right)+q_{2} \Psi\left(\frac{q_{1} b+a}{1+q_{1}}, c\right)+\Psi\left(\frac{q_{1} b+a}{1+q_{1}}, \frac{q_{2} d+c}{1+q_{2}}\right)}{\left(1+q_{1}\right)\left(1+q_{2}\right)} \text {. }
\end{aligned}
$$

- If $A=a, B=\frac{q_{1} a+b}{1+q_{1}}, C=c, D=\frac{q_{2} c+d}{1+q_{2}}$, then

$$
\begin{aligned}
\Psi( & \left.\frac{\left(2 q_{1}+q_{1}^{2}\right) a+b}{\left(1+q_{1}\right)^{2}}, \frac{\left(2 q_{2}+q_{2}^{2}\right) c+d}{\left(1+q_{2}\right)^{2}}\right) \\
\leq & \frac{1+q_{1}}{2(b-a)} \int_{a}^{\frac{q_{1} a+b}{1+q_{1}}} \Psi\left(u, \frac{\left(2 q_{2}+q_{2}^{2}\right) c+d}{\left(1+q_{2}\right)^{2}}\right){ }_{a} d_{q_{1}} u \\
& +\frac{1+q_{2}}{2(d-c)} \int_{c}^{\frac{q_{2} c+d}{1+q_{2}}} \Psi\left(\frac{\left(2 q_{1}+q_{1}^{2}\right) a+b}{\left(1+q_{1}\right)^{2}}, v\right){ }_{c} d_{q_{2}} v \\
\leq & \frac{\left(1+q_{1}\right)\left(1+q_{2}\right)}{(b-a)(d-c)} \int_{a}^{\frac{q_{1} a+b}{1+q_{1}}} \int_{c}^{\frac{q_{2} c+d}{1+q_{2}}} f(u, v)_{c} d_{q_{2}} v_{a} d_{q_{1}} u \\
\leq & \frac{q_{2}\left(1+q_{1}\right)}{2\left(1+q_{2}\right)(b-a)} \int_{a}^{\frac{q_{1} a+b}{1+q_{1}}} \Psi(u, c){ }_{a} d_{q_{1}} u \\
& +\frac{1+q_{1}}{2\left(1+q_{2}\right)(b-a)} \int_{a}^{\frac{q_{1} a+b}{1+q_{1}}} \Psi\left(u, \frac{q_{2} d+c}{1+q_{2}}\right){ }_{a} d_{q_{1}} u \\
& +\frac{q_{1}\left(1+q_{2}\right)}{2\left(1+q_{1}\right)(d-c)} \int_{c}^{\frac{q_{2} c+d}{1+q_{2}}} \Psi(a, v)_{c} d_{q_{2}} v \\
& +\frac{1+q_{2}}{2\left(1+q_{1}\right)(d-c)} \int_{c}^{\frac{q_{2} c+d}{1+q_{2}}} \Psi\left(\frac{q_{1} a+b}{1+q_{1}}, v\right){ }_{c} d_{q_{2}} v
\end{aligned}
$$




$$
\leq \frac{q_{1} q_{2} \Psi(a, c)+q_{1} \Psi\left(a, \frac{q_{2} c+d}{1+q_{2}}\right)+q_{2} \Psi\left(\frac{q_{1} a+b}{1+q_{1}}, c\right)+\Psi\left(\frac{q_{1} a+b}{1+q_{1}}, \frac{q_{2} c+d}{1+q_{2}}\right)}{\left(1+q_{1}\right)\left(1+q_{2}\right)}
$$

- If $A=a, B=\frac{q_{1} b+a}{1+q_{1}}, C=c, D=\frac{q_{2} c+d}{1+q_{2}}$, then

$$
\begin{aligned}
\Psi( & \left.\frac{\left(1+q_{1}+q_{1}^{2}\right) a+q_{1} b}{\left(1+q_{1}\right)^{2}}, \frac{\left(2 q_{2}+q_{2}^{2}\right) c+d}{\left(1+q_{2}\right)^{2}}\right) \\
\leq & \frac{1+q_{1}}{2 q_{1}(b-a)} \int_{a}^{\frac{q_{1} b+a}{1+q_{1}}} \Psi\left(u, \frac{\left(2 q_{2}+q_{2}^{2}\right) c+d}{\left(1+q_{2}\right)^{2}}\right){ }_{a} d_{q_{1}} u \\
& +\frac{1+q_{2}}{2(d-c)} \int_{c}^{\frac{q_{2} c+d}{1+q_{2}}} \Psi\left(\frac{\left(1+q_{1}+q_{1}^{2}\right) a+q_{1} b}{\left(1+q_{1}\right)^{2}}, v\right){ }_{c} d_{q_{2}} v \\
\leq & \frac{\left(1+q_{1}\right)\left(1+q_{2}\right)}{q_{1}(b-a)(d-c)} \int_{a}^{\frac{q_{1} b+a}{1+q_{1}}} \int_{c}^{\frac{q_{2} c+d}{1+q_{2}}} \Psi(u, v){ }_{c} d_{q_{2}} v_{a} d_{q_{1}} u \\
\leq & \frac{q_{2}\left(1+q_{1}\right)}{2 q_{1}\left(1+q_{2}\right)(b-a)} \int_{a}^{\frac{q_{1} b+a}{1+q_{1}}} \Psi(u, c){ }_{a} d_{q_{1}} u \\
& +\frac{1+q_{1}}{2 q_{1}\left(1+q_{2}\right)(b-a)} \int_{a}^{\frac{q_{1} b+a}{1+q_{1}}} \Psi\left(u, \frac{q_{2} d+c}{1+q_{2}}\right){ }_{a} d_{q_{1}} u \\
& +\frac{q_{1}\left(1+q_{2}\right)}{2\left(1+q_{1}\right)(d-c)} \int_{c}^{\frac{q_{2} c+d}{1+q_{2}}} \Psi(a, v)_{c} d_{q_{2}} v \\
& +\frac{1+q_{2}}{2\left(1+q_{1}\right)(d-c)} \int_{c}^{\frac{q_{2} c+d}{1+q_{2}}} \Psi\left(\frac{q_{1} b+a}{1+q_{1}}, v\right){ }_{c} d_{q_{2}} v \\
& \frac{q_{1} q_{2}(a, c)+q_{1} \Psi\left(a, \frac{q_{2} c+d}{1+q_{2}}\right)+q_{2} \Psi\left(\frac{q_{1} b+a}{1+q_{1}}, c\right)+\Psi\left(\frac{q_{1} b+a}{1+q_{1}}, \frac{q_{2} c+d}{1+q_{2}}\right)}{\left(1+q_{1}\right)\left(1+q_{2}\right)}
\end{aligned}
$$

In view of the above results and literatures, and following this tendency of the newly introduced $q$-derivatives and $q$-integrals, the aim of this paper is to establish some new refinements of the $H-H$ inequality in the quantum domain using coordinated convex functions. Several special cases from our main results will be given in detail and many well-known results will be recaptured. At the end, we provide a briefly conclusion as well.

\section{Main results}

By utilizing Theorem 1.3, we have the new result.

Theorem 2.1 Suppose that $\Psi: \Delta \rightarrow \mathbb{R}_{e}$ is a coordinated convex function on $\Delta$ and $\Psi \in$ $L_{1}(\Delta)$. Then we have

$$
\begin{aligned}
& \Psi\left(\frac{A+q_{1} B}{1+q_{1}}, \frac{C+q_{2} D}{1+q_{2}}\right) \\
& \quad \leq \frac{1}{2}\left[\frac{1}{B-A} \int_{A}^{B} \Psi\left(u, \frac{C+q_{2} D}{1+q_{2}}\right)^{B} d_{q_{1}} u+\frac{1}{D-C} \int_{C}^{D} \Psi\left(\frac{A+q_{1} B}{1+q_{1}}, v\right)^{D} d_{q_{2}} v\right] \\
& \quad \leq \frac{1}{(B-A)(D-C)} \int_{A}^{B} \int_{C}^{D} \Psi(u, v)^{D} d_{q_{2}} v^{B} d_{q_{1}} u
\end{aligned}
$$




$$
\begin{aligned}
\leq & \frac{1}{2\left(1+q_{2}\right)(B-A)} \int_{A}^{B} \Psi(u, C)^{B} d_{q_{1}} u+\frac{q_{2}}{2\left(1+q_{2}\right)(B-A)} \int_{A}^{B} \Psi(u, D)^{B} d_{q_{1}} u \\
& +\frac{1}{2\left(1+q_{1}\right)(D-C)} \int_{C}^{D} \Psi(A, v)^{D} d_{q_{2}} v+\frac{q_{1}}{2\left(1+q_{1}\right)(D-C)} \int_{C}^{D} \Psi(B, v)^{D} d_{q_{2}} v \\
\leq & \frac{\Psi(A, C)+q_{2} \Psi(A, D)+q_{1} \Psi(B, C)+q_{1} q_{2} \Psi(B, D)}{\left(1+q_{1}\right)\left(1+q_{2}\right)} .
\end{aligned}
$$

Proof Due to the coordinated convexity of $\Psi: \Delta \rightarrow \mathbb{R}_{e}$, the partial mapping $\Psi_{u}:[C, D] \rightarrow$ $\mathbb{R}_{e}$ defined by $\Psi_{u}(y)=\Psi(u, y)$ for all $u \in[A, B]$ will be convex on $[C, D]$. Analogously, $\Psi_{v}$ : $[A, B] \rightarrow \mathbb{R}_{e}$ defined by $\Psi_{v}(x)=\Psi(x, v)$ for all $v \in[C, D]$ is convex on $[A, B]$. Then, by the Theorem 1.3, we get

$$
\Psi_{u}\left(\frac{C+q_{2} D}{1+q_{2}}\right) \leq \frac{1}{D-C} \int_{C}^{D} \Psi_{u}(v)^{D} d_{q_{2}} v \leq \frac{\Psi_{u}(C)+q_{2} \Psi_{u}(D)}{1+q_{2}},
$$

or

$$
\Psi\left(u, \frac{C+q_{2} D}{1+q_{2}}\right) \leq \frac{1}{D-C} \int_{C}^{D} \Psi(u, v)^{D} d_{q_{2}} v \leq \frac{\Psi(u, C)+q_{2} \Psi(u, D)}{1+q_{2}} .
$$

Integrating the inequality over $[A, B]$, we have

$$
\begin{aligned}
\frac{1}{B-A} \int_{A}^{B} \Psi\left(u, \frac{C+q_{2} D}{1+q_{2}}\right){ }^{B} d_{q_{1}} u \leq & \frac{1}{(B-A)(D-C)} \int_{A}^{B} \int_{C}^{D} \Psi(u, v)^{D} d_{q_{2}} v^{B} d_{q_{1}} u \\
\leq & \frac{1}{\left(1+q_{2}\right)(B-A)} \int_{A}^{B} \Psi(u, C)^{B} d_{q_{1}} u \\
& +\frac{q_{2}}{\left(1+q_{2}\right)(B-A)} \int_{A}^{B} \Psi(u, D)^{B} d_{q_{1}} u .
\end{aligned}
$$

Now, by the convexity of $\Psi_{v}$, by the Theorem 1.3 , we have

$$
\Psi_{\nu}\left(\frac{A+q_{1} B}{1+q_{1}}\right) \leq \frac{1}{B-A} \int_{A}^{B} \Psi_{\nu}(u)^{B} d_{q_{1}} u \leq \frac{\Psi_{v}(A)+q_{1} \Psi_{\nu}(B)}{1+q_{1}},
$$

or

$$
\Psi\left(\frac{A+q_{1} B}{1+q_{1}}, v\right) \leq \frac{1}{B-A} \int_{A}^{B} \Psi(u, v){ }^{B} d_{q_{1}} u \leq \frac{\Psi(A, v)+q_{1} \Psi(B, v)}{1+q_{1}} .
$$

Evaluating the average integral over $[C, D]$, we have

$$
\begin{aligned}
& \frac{1}{D-C} \int_{C}^{D} \Psi\left(\frac{A+q_{1} B}{1+q_{2}}, v\right)^{D} d_{q_{2}} v \\
& \quad \leq \frac{1}{(B-A)(D-C)} \int_{A}^{B} \int_{C}^{D} \Psi(u, v)^{D} d_{q_{2}} v^{B} d_{q_{1}} u \\
& \quad \leq \frac{1}{\left(1+q_{1}\right)(D-C)} \int_{C}^{D} \Psi(A, v)^{D} d_{q_{2}} v+\frac{q_{1}}{\left(1+q_{1}\right)(D-C)} \int_{C}^{D} \Psi(B, v)^{D} d_{q_{2}} v .
\end{aligned}
$$


Adding (2.5) and (2.6), we get

$$
\begin{aligned}
& \frac{1}{B-A} \int_{A}^{B} \Psi\left(u, \frac{C+q_{2} D}{1+q_{2}}\right){ }^{B} d_{q_{1}} u+\frac{1}{D-C} \int_{C}^{D} \Psi\left(\frac{A+q_{1} B}{1+q_{2}}, v\right)^{D} d_{q_{2}} v \\
& \leq \frac{2}{(B-A)(D-C)} \int_{A}^{B} \int_{C}^{D} \Psi(u, v)^{D} d_{q_{2}} v^{B} d_{q_{1}} u \\
& \leq \frac{1}{\left(1+q_{2}\right)(B-A)} \int_{A}^{B} \Psi(u, C)^{B} d_{q_{1}} u+\frac{q_{2}}{\left(1+q_{2}\right)(B-A)} \int_{A}^{B} \Psi(u, D)^{B} d_{q_{1}} u \\
& \quad+\frac{1}{\left(1+q_{1}\right)(D-C)} \int_{C}^{D} \Psi(A, v)^{D} d_{q_{2}} v+\frac{q_{1}}{\left(1+q_{1}\right)(D-C)} \int_{C}^{D} \Psi(B, v)^{D} d_{q_{2}} v .
\end{aligned}
$$

Again, by the convexity and applying the first inequality of Theorem 1.3, we have

$$
\Psi\left(\frac{A+q_{1} B}{1+q_{2}}, \frac{C+q_{2} D}{1+q_{2}}\right) \leq \frac{1}{D-C} \int_{C}^{D} \Psi\left(\frac{A+q_{1} B}{1+q_{2}}, v\right){ }^{D} d_{q_{2}} v
$$

and

$$
\Psi\left(\frac{A+q_{1} B}{1+q_{2}}, \frac{C+q_{2} D}{1+q_{2}}\right) \leq \frac{1}{B-A} \int_{A}^{B} \Psi\left(u, \frac{C+q_{2} D}{1+q_{2}}\right){ }^{B} d_{q_{1}} u
$$

Similarly, applying the second inequality of Theorem 1.3 and convexity of $\Psi_{u}$ and $\Psi_{v}$, we have

$$
\begin{aligned}
& \frac{1}{\left(1+q_{2}\right)(B-A)} \int_{A}^{B} \Psi(u, C)^{B} d_{q_{1}} u+\frac{q_{2}}{\left(1+q_{2}\right)(B-A)} \int_{A}^{B} \Psi(u, D)^{B} d_{q_{1}} u \\
& \quad+\frac{1}{\left(1+q_{1}\right)(D-C)} \int_{C}^{D} \Psi(A, v)^{D} d_{q_{2}} v+\frac{q_{1}}{\left(1+q_{1}\right)(D-C)} \int_{C}^{D} \Psi(B, v)^{D} d_{q_{2}} v \\
& \leq \frac{2}{\left(1+q_{1}\right)\left(1+q_{2}\right)}\left[\Psi(A, C)+q_{2} \Psi(A, D)+q_{1} \Psi(B, C)+q_{1} q_{2} \Psi(B, D)\right] .
\end{aligned}
$$

Combining inequalities (2.7), (2.8), (2.9) and (2.10), we directly obtain our desired inequality.

Remark 2.1 From Theorem 2.1, we can deduce the following midpoint special cases.

- If $A=\frac{q_{1} a+b}{1+q_{1}}, B=b, C=\frac{q_{2} d+c}{1+q_{2}}, D=d$, then

$$
\begin{aligned}
\Psi( & \left.\frac{q_{1} a+\left(1+q_{1}+q_{1}^{2}\right) b}{\left(1+q_{1}\right)^{2}}, \frac{c+\left(2 q_{2}+q_{2}^{2}\right) d}{\left(1+q_{2}\right)^{2}}\right) \\
\leq & \frac{1+q_{1}}{2 q_{1}(b-a)} \int_{\frac{q_{1} a+b}{1+q_{1}}}^{b} \Psi\left(u, \frac{c+\left(2 q_{2}+q_{2}^{2}\right) d}{\left(1+q_{2}\right)^{2}}\right){ }^{b} d_{q_{1}} u \\
& +\frac{1+q_{2}}{2(d-c)} \int_{\frac{q_{2} d+c}{1+q_{2}}}^{d} \Psi\left(\frac{q_{1} a+\left(1+q_{1}+q_{1}^{2}\right) b}{\left(1+q_{1}\right)^{2}}, v\right) d d_{q_{2}} v \\
\leq & \frac{\left(1+q_{1}\right)\left(1+q_{2}\right)}{q_{1}(b-a)(d-c)} \int_{\frac{q_{1} a+b}{1+q_{1}}}^{b} \int_{\frac{q_{2} d+c}{1+q_{2}}}^{d} \Psi(u, v)^{d} d_{q_{2}} v^{b} d_{q_{1}} u \\
\leq & \frac{1+q_{1}}{2 q_{1}\left(1+q_{2}\right)(b-a)} \int_{\frac{q_{1} a+b}{1+q_{1}}}^{b} \Psi\left(u, \frac{q_{2} d+c}{1+q_{2}}\right){ }^{b} d_{q_{1}} u
\end{aligned}
$$


Alqudah et al. Advances in Difference Equations

(2021) 2021:264

Page 12 of 29

$$
\begin{aligned}
& +\frac{q_{2}\left(1+q_{1}\right)}{2 q_{1}\left(1+q_{2}\right)(b-a)} \int_{\frac{q_{1} a+b}{1+q_{1}}}^{b} \Psi(u, d)^{b} d_{q_{1}} u \\
& \left.+\frac{1+q_{2}}{2\left(1+q_{1}\right)(d-c)} \int_{\frac{q_{2} d+c}{1+q_{2}}}^{d} \Psi\left(\frac{q_{1} a+b}{1+q_{1}}, v\right)\right)^{d} d_{q_{2}} v \\
& +\frac{q_{1}\left(1+q_{2}\right)}{2\left(1+q_{1}\right)(d-c)} \int_{\frac{q_{2} d+c}{1+q_{2}}}^{d} \Psi(b, v)^{d} d_{q_{2}} v \\
& \leq \frac{\Psi\left(\frac{q_{1} a+b}{1+q_{1}}, \frac{q_{2} d+c}{1+q_{2}}\right)+q_{2} \Psi\left(\frac{q_{1} a+b}{1+q_{1}}, d\right)+q_{1} \Psi\left(b, \frac{q_{2} d+c}{1+q_{2}}\right)+q_{1} q_{2} \Psi(b, d)}{\left(1+q_{1}\right)\left(1+q_{2}\right)} .
\end{aligned}
$$

- If $A=\frac{q_{1} b+a}{1+q_{1}}, B=b, C=\frac{q_{2} d+c}{1+q_{2}}, D=d$, then

$$
\begin{aligned}
\Psi( & \left.\frac{a+\left(2 q_{1}+q_{1}^{2}\right) b}{\left(1+q_{1}\right)^{2}}, \frac{c+\left(2 q_{2}+q_{2}^{2}\right) d}{\left(1+q_{2}\right)^{2}}\right) \\
\leq & \frac{1+q_{1}}{2(b-a)} \int_{\frac{q_{1} b+a}{1+q_{1}}}^{b} \Psi\left(u, \frac{c+\left(2 q_{2}+q_{2}^{2}\right) d}{\left(1+q_{2}\right)^{2}}\right){ }^{b} d_{q_{1}} u \\
& +\frac{1+q_{2}}{2(d-c)} \int_{\frac{q_{2} d+c}{1+q_{2}}}^{d} \Psi\left(\frac{a+\left(2 q_{1}+q_{1}^{2}\right) b}{\left(1+q_{1}\right)^{2}}, v\right)^{d} d_{q_{2}} v \\
\leq & \frac{\left(1+q_{1}\right)\left(1+q_{2}\right)}{(b-a)(d-c)} \int_{\frac{q_{1} b+a}{1+q_{1}}}^{b} \int_{\frac{q_{2} d+c}{1+q_{2}}}^{d} \Psi(u, v){ }^{d} d_{q_{2}} v^{b} d_{q_{1}} u \\
\leq & \frac{1+q_{1}}{2\left(1+q_{2}\right)(b-a)} \int_{\frac{q_{1} b+a}{1+q_{1}}}^{b} \Psi\left(u, \frac{q_{2} d+c}{1+q_{2}}\right){ }^{b} d_{q_{1}} u \\
& +\frac{q_{2}\left(1+q_{1}\right)}{2\left(1+q_{2}\right)(b-a)} \int_{\frac{q_{1} b+a}{1+q_{1}}}^{b} \Psi(u, d)^{b} d_{q_{1}} u \\
& +\frac{1+q_{2}}{2\left(1+q_{1}\right)(d-c)} \int_{\frac{q_{2} d+c}{1+q_{2}}}^{d} \Psi\left(\frac{q_{1} b+a}{1+q_{1}}, v\right){ }^{d} d_{q_{2}} v \\
\quad & \frac{\Psi\left(\frac{q_{1} b+a}{1+q_{1}}, \frac{q_{2} d+c}{1+q_{2}}\right)+q_{2} \Psi\left(\frac{q_{1} b+a}{1+q_{1}}, d\right)+q_{1} \Psi\left(b, \frac{q_{2} d+c}{1+q_{2}}\right)+q_{1} q_{2} \Psi(b, d)}{\left(1+q_{2}\right)} \int_{\frac{q_{2} d+c}{1+q_{2}}}^{d} \Psi(b, v)^{d} d_{q_{2}} v
\end{aligned}
$$

- If $A=\frac{q_{1} a+b}{1+q_{1}}, B=b, C=\frac{q_{2} c+d}{1+q_{2}}, D=d$, then

$$
\begin{gathered}
\Psi\left(\frac{q_{1} a+\left(1+q_{1}+q_{1}^{2}\right) b}{\left(1+q_{1}\right)^{2}}, \frac{q_{2} c+\left(1+q_{2}+q_{2}^{2}\right) d}{\left(1+q_{2}\right)^{2}}\right) \\
\leq \frac{1+q_{1}}{2 q_{1}(b-a)} \int_{\frac{q_{1} a+b}{1+q_{1}}}^{b} \Psi\left(u, \frac{c+\left(2 q_{2}+q_{2}^{2}\right) d}{\left(1+q_{2}\right)^{2}}\right){ }^{b} d_{q_{1}} u \\
\quad+\frac{1+q_{2}}{2 q_{2}(d-c)} \int_{\frac{q_{2} c+d}{1+q_{2}}}^{d} \Psi\left(\frac{q_{1} a+\left(1+q_{1}+q_{1}^{2}\right) b}{\left(1+q_{1}\right)^{2}}, v\right) d^{d} d_{q_{2}} v \\
\leq \frac{\left(1+q_{1}\right)\left(1+q_{2}\right)}{q_{1} q_{2}(b-a)(d-c)} \int_{\frac{q_{1} a+b}{1+q_{1}}}^{b} \int_{\frac{q_{2} c+d}{1+q_{2}}}^{d} \Psi(u, v)^{d} d_{q_{2}} v^{b} d_{q_{1}} u
\end{gathered}
$$




$$
\begin{aligned}
\leq & \frac{1+q_{1}}{2 q_{1}\left(1+q_{2}\right)(b-a)} \int_{\frac{q_{1} a+b}{1+q_{1}}}^{b} \Psi\left(u, \frac{q_{2} d+c}{1+q_{2}}\right){ }^{b} d_{q_{1}} u \\
& +\frac{q_{2}\left(1+q_{1}\right)}{2 q_{1}\left(1+q_{2}\right)(b-a)} \int_{\frac{q_{1} a+b}{1+q_{1}}}^{b} \Psi(u, d)^{b} d_{q_{1}} u \\
& +\frac{1+q_{2}}{2 q_{2}\left(1+q_{1}\right)(d-c)} \int_{\frac{q_{2} c+d}{1+q_{2}}}^{d} \Psi\left(\frac{q_{1} a+b}{1+q_{1}}, v\right){ }^{d} d_{q_{2}} v \\
& +\frac{q_{1}\left(1+q_{2}\right)}{2 q_{2}\left(1+q_{1}\right)(d-c)} \int_{\frac{q_{2} c+d}{1+q_{2}}}^{d} \Psi(b, v)^{d} d_{q_{2}} v \\
\leq & \frac{\Psi\left(\frac{q_{1} a+b}{1+q_{1}}, \frac{q_{2} c+d}{1+q_{2}}\right)+q_{2} \Psi\left(\frac{q_{1} a+b}{1+q_{1}}, d\right)+q_{1} \Psi\left(b, \frac{q_{2} c+d}{1+q_{2}}\right)+q_{1} q_{2} \Psi(b, d)}{\left(1+q_{1}\right)\left(1+q_{2}\right)} .
\end{aligned}
$$

- If $A=\frac{q_{1} b+a}{1+q_{1}}, B=b, C=\frac{q_{2} c+d}{1+q_{2}}, D=d$, then

$$
\begin{aligned}
\Psi( & \left.\frac{a+\left(2 q_{1}+q_{1}^{2}\right) b}{\left(1+q_{1}\right)^{2}}, \frac{q_{2} c+\left(1+q_{2}+q_{2}^{2}\right) d}{\left(1+q_{2}\right)^{2}}\right) \\
\leq & \frac{1+q_{1}}{2(b-a)} \int_{\frac{q_{1} b+a}{1+q_{1}}}^{b} \Psi\left(u, \frac{q_{2} c+\left(1+q_{2}+q_{2}^{2}\right) d}{\left(1+q_{2}\right)^{2}}\right){ }^{b} d_{q_{1}} u \\
& +\frac{1+q_{2}}{2 q_{2}(d-c)} \int_{\frac{q_{2} c+d}{1+q_{2}}}^{d} \Psi\left(\frac{a+\left(2 q_{1}+q_{1}^{2}\right) b}{\left(1+q_{1}\right)^{2}}, v\right){ }^{d} d_{q_{2}} v \\
\leq & \frac{\left(1+q_{1}\right)\left(1+q_{2}\right)}{q_{2}(b-a)(d-c)} \int_{\frac{q_{1} b+a}{1+q_{1}}}^{b} \int_{\frac{q_{2} c+d}{1+q_{2}}}^{d} \Psi(u, v)^{d} d_{q_{2}} v^{b} d_{q_{1}} u \\
\leq & \frac{1+q_{1}}{2\left(1+q_{2}\right)(b-a)} \int_{\frac{q_{1} b+a}{1+q_{1}}}^{b} \Psi\left(u, \frac{q_{2} d+c}{1+q_{2}}\right){ }^{b} d_{q_{1}} u \\
& +\frac{q_{2}\left(1+q_{1}\right)}{2\left(1+q_{2}\right)(b-a)} \int_{\frac{q_{1} b+a}{1+q_{1}}}^{b} \Psi(u, d)^{b} d_{q_{1}} u \\
& +\frac{1+q_{2}}{2 q_{2}\left(1+q_{1}\right)(d-c)} \int_{\frac{q_{2} c+d}{1+q_{2}}}^{d} \Psi\left(\frac{q_{1} b+a}{1+q_{1}}, v\right) d d_{q_{2}} v \\
& +\frac{q_{1}\left(1+q_{2}\right)}{2 q_{2}\left(1+q_{1}\right)(d-c)} \int_{\frac{q_{2} c+d}{1+q_{2}}}^{d} \Psi(b, v)^{d} d_{q_{2}} v \\
\leq & \frac{\Psi\left(\frac{q_{1} b+a}{1+q_{1}}, \frac{q_{2} c+d}{1+q_{2}}\right)+q_{2} \Psi\left(\frac{q_{1} b+a}{1+q_{1}}, d\right)+q_{1} \Psi\left(b, \frac{q_{2} c+d}{1+q_{2}}\right)+q_{1} q_{2} \Psi(b, d)}{\left(1+q_{1}\right)\left(1+q_{2}\right)} .
\end{aligned}
$$

The application of the Theorems 1.2 and 1.3 leads to the following result.

Theorem 2.2 Suppose that $\Psi: \Delta \rightarrow \mathbb{R}_{e}$ is a coordinated convex function on $\Delta$ and $\Psi \in$ $L_{1}(\Delta)$. Then we have

$$
\begin{aligned}
& \Psi\left(\frac{A+q_{1} B}{1+q_{1}}, \frac{q_{2} C+D}{1+q_{2}}\right) \\
& \quad \leq \frac{1}{2}\left[\frac{1}{B-A} \int_{A}^{B} \Psi\left(u, \frac{q_{2} C+D}{1+q_{2}}\right){ }^{B} d_{q_{1}} u+\frac{1}{D-C} \int_{C}^{D} \Psi\left(\frac{A+q_{1} B}{1+q_{1}}, v\right){ }_{C} d_{q_{2}} v\right]
\end{aligned}
$$




$$
\begin{aligned}
\leq & \frac{1}{(B-A)(D-C)} \int_{A}^{B} \int_{C}^{D} \Psi(u, v)_{C} d_{q_{2}} v^{B} d_{q_{1}} u \\
\leq & {\left[\frac{q_{2}}{2\left(1+q_{2}\right)(B-A)} \int_{A}^{B} \Psi(u, C)^{B} d_{q_{1}} u+\frac{1}{2\left(1+q_{2}\right)(B-A)} \int_{A}^{B} \Psi(u, D)^{B} d_{q_{1}} u\right.} \\
& \left.+\frac{1}{2\left(1+q_{1}\right)(D-C)} \int_{C}^{D} \Psi(A, v)_{C} d_{q_{2}} v+\frac{q_{1}}{2\left(1+q_{1}\right)(D-C)} \int_{C}^{D} \Psi(B, v)_{C} d_{q_{2}} v\right] \\
\leq & \frac{q_{2} \Psi(A, C)+\Psi(A, D)+q_{1} q_{2} \Psi(B, C)+q_{1} \Psi(B, D)}{\left(1+q_{1}\right)\left(1+q_{2}\right)} .
\end{aligned}
$$

Proof The proof is omitted.

Remark 2.2 From Theorem 2.2, we can deduce the following midpoint special cases.

- If $A=\frac{q_{1} a+b}{1+q_{1}}, B=b, C=c, D=\frac{q_{2} d+c}{1+q_{2}}$, then

$$
\begin{aligned}
\Psi( & \left.\frac{q_{1} a+\left(1+q_{1}+q_{1}^{2}\right) b}{\left(1+q_{1}\right)^{2}}, \frac{\left(1+q_{2}+q_{2}^{2}\right) c+q_{2} d}{\left(1+q_{2}\right)^{2}}\right) \\
\leq & \frac{1+q_{1}}{2 q_{1}(b-a)} \int_{\frac{q_{1} a+b}{1+q_{1}}}^{b} \Psi\left(u, \frac{\left(1+q_{2}+q_{2}^{2}\right) c+q_{2} d}{\left(1+q_{2}\right)^{2}}\right){ }^{b} d_{q_{1}} u \\
& +\frac{1+q_{2}}{2 q_{2}(d-c)} \int_{c}^{\frac{q_{2} d+c}{1+q_{2}}} \Psi\left(\frac{q_{1} a+\left(1+q_{1}+q_{1}^{2}\right) b}{\left(1+q_{1}\right)^{2}}, v\right){ }_{c} d_{q_{2}} v \\
\leq & \frac{\left(1+q_{1}\right)\left(1+q_{2}\right)}{q_{1} q_{2}(b-a)(d-c)} \int_{\frac{q_{1} a+b}{1+q_{1}}}^{b} \int_{c}^{\frac{q_{2} d+c}{1+q_{2}}} \Psi(u, v)_{c} d_{q_{2}} v^{b} d_{q_{1}} u \\
\leq & \frac{q_{2}\left(1+q_{1}\right)}{2 q_{1}\left(1+q_{2}\right)(b-a)} \int_{\frac{q_{1} a+b}{1+q_{1}}}^{b} \Psi(u, c)^{b} d_{q_{1}} u \\
& +\frac{1+q_{1}}{2 q_{1}\left(1+q_{2}\right)(b-a)} \int_{\frac{q_{1} a+b}{1+q_{1}}}^{b} \Psi\left(u, \frac{q_{2} d+c}{1+q_{2}}\right){ }^{b} d_{q_{1}} u \\
& +\frac{1+q_{2}}{2 q_{2}\left(1+q_{1}\right)(d-c)} \int_{c}^{\frac{q_{2} d d c}{1+q_{2}}} \Psi\left(\frac{q_{1} a+b}{1+q_{1}}, v\right){ }_{c} d_{q_{2}} v \\
& +\frac{q_{1}\left(1+q_{2}\right)}{2 q_{2}\left(1+q_{1}\right)(d-c)} \int_{c}^{\frac{q_{2} d+c}{1+q_{2}}} \Psi(b, v)_{c} d_{q_{2}} v \\
\leq & \frac{q_{2} \Psi\left(\frac{q_{1} a+b}{1+q_{1}}, c\right)+\Psi\left(\frac{q_{1} a+b}{1+q_{1}}, \frac{q_{2} d+c}{1+q_{2}}\right)+q_{1} q_{2} \Psi(b, c)+q_{1} \Psi\left(b, \frac{q_{2} d+c}{1+q_{2}}\right)}{\left(1+q_{1}\right)\left(1+q_{2}\right)} .
\end{aligned}
$$

- If $A=\frac{q_{1} b+a}{1+q_{1}}, B=b, C=c, D=\frac{q_{2} d+c}{1+q_{2}}$, then

$$
\begin{aligned}
\Psi( & \left.\frac{a+\left(2 q_{1}+q_{1}^{2}\right) b}{\left(1+q_{1}\right)^{2}}, \frac{\left(1+q_{2}+q_{2}^{2}\right) c+q_{2} d}{\left(1+q_{2}\right)^{2}}\right) \\
\leq & \frac{1+q_{1}}{2(b-a)} \int_{\frac{q_{1} b+a}{1+q_{1}}}^{b} \Psi\left(u, \frac{\left(1+q_{2}+q_{2}^{2}\right) c+q_{2} d}{\left(1+q_{2}\right)^{2}}\right){ }^{b} d_{q_{1}} u \\
& +\frac{1+q_{2}}{2 q_{2}(d-c)} \int_{c}^{\frac{q_{2} d+c}{1+q_{2}}} \Psi\left(\frac{a+\left(2 q_{1}+q_{1}^{2}\right) b}{\left(1+q_{1}\right)^{2}}, v\right){ }_{c} d_{q_{2}} v
\end{aligned}
$$




$$
\begin{aligned}
\leq & \frac{\left(1+q_{1}\right)\left(1+q_{2}\right)}{q_{2}(b-a)(d-c)} \int_{\frac{q_{1} b+a}{1+q_{1}}}^{b} \int_{c}^{\frac{q_{2} d+c}{1+q_{2}}} \Psi(u, v)_{c} d_{q_{2}} v^{b} d_{q_{1}} u \\
\leq & \frac{q_{2}\left(1+q_{1}\right)}{2\left(1+q_{2}\right)(b-a)} \int_{\frac{q_{1} b+a}{1+q_{1}}}^{b} \Psi(u, c)^{b} d_{q_{1}} u \\
& +\frac{1+q_{1}}{2\left(1+q_{2}\right)(b-a)} \int_{\frac{q_{1} b+a}{1+q_{1}}}^{b} \Psi\left(u, \frac{q_{2} d+c}{1+q_{2}}\right){ }^{b} d_{q_{1}} u \\
& +\frac{1+q_{2}}{2 q_{2}\left(1+q_{1}\right)(d-c)} \int_{c}^{\frac{q_{2} d+c}{1+q_{2}}} \Psi\left(\frac{q_{1} b+a}{1+q_{1}}, v\right){ }_{c} d_{q_{2}} v \\
& +\frac{q_{1}\left(1+q_{2}\right)}{2 q_{2}\left(1+q_{1}\right)(d-c)} \int_{c}^{\frac{q_{2} d+c}{1+q_{2}}} \Psi(b, v)_{c} d_{q_{2}} v \\
\leq & \frac{q_{2} \Psi\left(\frac{q_{1} b+a}{1+q_{1}}, c\right)+\Psi\left(\frac{q_{1} b+a}{1+q_{1}}, \frac{q_{2} d+c}{1+q_{2}}\right)+q_{1} q_{2} \Psi(b, c)+q_{1} \Psi\left(b, \frac{q_{2} d+c}{1+q_{2}}\right)}{\left(1+q_{1}\right)\left(1+q_{2}\right)} .
\end{aligned}
$$

- If $A=\frac{q_{1} a+b}{1+q_{1}}, B=b, C=c, D=\frac{q_{2} c+d}{1+q_{2}}$, then

$$
\begin{aligned}
\Psi( & \left.\frac{q_{1} a+\left(1+q_{1}+q_{1}^{2}\right) b}{\left(1+q_{1}\right)^{2}}, \frac{c\left(2 q_{2}+q_{2}^{2}\right)+d}{\left(1+q_{2}\right)^{2}}\right) \\
\leq & \frac{1+q_{1}}{2 q_{1}(b-a)} \int_{\frac{q_{1} a+b}{1+q_{1}}}^{b} \Psi\left(u, \frac{c\left(2 q_{2}+q_{2}^{2}\right)+d}{\left(1+q_{2}\right)^{2}}\right){ }^{b} d_{q_{1}} u \\
& +\frac{1+q_{2}}{2(d-c)} \int_{c}^{\frac{q_{2} c+d}{1+q_{2}}} \Psi\left(\frac{q_{1} a+\left(1+q_{1}+q_{1}^{2}\right) b}{\left(1+q_{1}\right)^{2}}, v\right){ }_{c} d_{q_{2}} v \\
\leq & \frac{\left(1+q_{1}\right)\left(1+q_{2}\right)}{q_{1}(b-a)(d-c)} \int_{\frac{q_{1} a+b}{1+q_{1}}}^{b} \int_{c}^{\frac{q_{2} c+d}{1+q_{2}}} \Psi(u, v)_{c} d_{q_{2}} v^{b} d_{q_{1}} u \\
\leq & \frac{q_{2}\left(1+q_{1}\right)}{2 q_{1}\left(1+q_{2}\right)(b-a)} \int_{\frac{q_{1} a+b}{1+q_{1}}}^{b} \Psi(u, c)^{b} d_{q_{1}} u \\
& +\frac{1+q_{1}}{2 q_{1}\left(1+q_{2}\right)(b-a)} \int_{\frac{q_{1} a+b}{1+q_{1}}}^{b} \Psi\left(u, \frac{q_{2} d+c}{1+q_{2}}\right){ }^{b} d_{q_{1}} u \\
& +\frac{1+q_{2}}{2\left(1+q_{1}\right)(d-c)} \int_{c}^{\frac{q_{2} c+d}{1+q_{2}}} \Psi\left(\frac{q_{1} a+b}{1+q_{1}}, v\right){ }_{c} d_{q_{2}} v \\
& +\frac{q_{1}\left(1+q_{2}\right)}{2\left(1+q_{1}\right)(d-c)} \int_{c}^{\frac{q_{2} c+d}{1+q_{2}}} \Psi(b, v)_{c} d_{q_{2}} v \\
\leq & \frac{q_{2} \Psi\left(\frac{q_{1} a+b}{1+q_{1}}, c\right)+\Psi\left(\frac{q_{1} a+b}{1+q_{1}}, \frac{q_{2} c+d}{1+q_{2}}\right)+q_{1} q_{2} \Psi(b, c)+q_{1} \Psi\left(b, \frac{q_{2} c+d}{1+q_{2}}\right)}{\left(1+q_{1}\right)\left(1+q_{2}\right)} .
\end{aligned}
$$

- If $A=\frac{q_{1} b+a}{1+q_{1}}, B=b, C=c, D=\frac{q_{2} c+d}{1+q_{2}}$, then

$$
\begin{aligned}
& \Psi\left(\frac{a+\left(2 q_{1}+q_{1}^{2}\right) b}{\left(1+q_{1}\right)^{2}}, \frac{\left(2 q_{2}+q_{2}^{2}\right) c+d}{\left(1+q_{2}\right)^{2}}\right) \\
& \quad \leq \frac{1+q_{1}}{2(b-a)} \int_{\frac{q_{1} b+a}{1+q_{1}}}^{b} \Psi\left(u, \frac{\left(2 q_{2}+q_{2}^{2}\right) c+d}{\left(1+q_{2}\right)^{2}}\right)^{b} d_{q_{1}} u
\end{aligned}
$$




$$
\begin{aligned}
& +\frac{1+q_{2}}{2(d-c)} \int_{c}^{\frac{q_{2} c+d}{1+q_{2}}} \Psi\left(\frac{a+\left(2 q_{1}+q_{1}^{2}\right) b}{\left(1+q_{1}\right)^{2}}, v\right){ }_{c} d_{q_{2}} v \\
\leq & \frac{\left(1+q_{1}\right)\left(1+q_{2}\right)}{(b-a)(d-c)} \int_{\frac{q_{1} b+a}{1+q_{1}}}^{b} \int_{c}^{\frac{q_{2} c+d}{1+q_{2}}} \Psi(u, v)_{c} d_{q_{2}} v^{b} d_{q_{1}} u \\
\leq & \frac{q_{2}\left(1+q_{1}\right)}{2\left(1+q_{2}\right)(b-a)} \int_{\frac{q_{1} b+a}{1+q_{1}}}^{b} \Psi(u, c)^{b} d_{q_{1}} u \\
& +\frac{1+q_{1}}{2\left(1+q_{2}\right)(b-a)} \int_{\frac{q_{1} b+a}{1+q_{1}}}^{b} \Psi\left(u, \frac{q_{2} d+c}{1+q_{2}}\right){ }^{b} d_{q_{1}} u \\
& +\frac{1+q_{2}}{2\left(1+q_{1}\right)(d-c)} \int_{c}^{\frac{q_{2} c+d}{1+q_{2}}} \Psi\left(\frac{q_{1} b+a}{1+q_{1}}, v\right){ }_{c} d_{q_{2}} v \\
& +\frac{q_{1}\left(1+q_{2}\right)}{2\left(1+q_{1}\right)(d-c)} \int_{c}^{\frac{q_{2} c+d}{1+q_{2}}} \Psi(b, v)_{c} d_{q_{2}} v \\
\leq & \frac{q_{2} \Psi\left(\frac{q_{1} b+a}{1+q_{1}}, c\right)+\Psi\left(\frac{q_{1} b+a}{1+q_{1}}, \frac{q_{2} c+d}{1+q_{2}}\right)+q_{1} q_{2} \Psi(b, c)+q_{1} \Psi\left(b, \frac{q_{2} c+d}{1+q_{2}}\right)}{\left(1+q_{1}\right)\left(1+q_{2}\right)} .
\end{aligned}
$$

Finally, we have the following inequalities by the utilizing Theorems 1.2 and 1.3.

Theorem 2.3 Let $\Psi: \Delta \rightarrow \mathbb{R}_{e}$ be a coordinated convex function on $\Delta$ and $q_{1}, q_{2} \in(0,1)$.

Then one has

$$
\begin{aligned}
\Psi( & \left.\frac{q_{1} A+B}{1+q_{1}}, \frac{C+q_{2} D}{1+q_{2}}\right) \\
\leq & \frac{1}{2}\left[\frac{1}{B-A} \int_{A}^{B} \Psi\left(u, \frac{C+q_{2} D}{1+q_{2}}\right){ }_{A} d_{q_{1}} u\right. \\
& \left.+\frac{1}{D-C} \int_{C}^{D} \Psi\left(\frac{q_{1} A+B}{1+q_{1}}, v\right){ }^{D} d_{q_{2}} v\right] \\
\leq & \frac{1}{(B-A)(D-C)} \int_{A}^{B} \int_{C}^{D} \Psi(u, v)^{D} d_{q_{2}} v_{A} d_{q_{1}} u \\
\leq & {\left[\frac{1}{2\left(1+q_{2}\right)(B-A)} \int_{A}^{B} \Psi(u, C)_{A} d_{q_{1}} u\right.} \\
& +\frac{q_{2}}{2\left(1+q_{2}\right)(B-A)} \int_{A}^{B} \Psi(u, D)_{A} d_{q_{1}} u \\
& +\frac{q_{1}}{2\left(1+q_{1}\right)(D-C)} \int_{C}^{D} \Psi(A, v)^{D} d_{q_{2}} v \\
& \left.+\frac{1}{2\left(1+q_{1}\right)(D-C)} \int_{C}^{D} \Psi(B, v)^{D} d_{q_{2}} v\right] \\
\leq & \frac{q_{1} \Psi(A, C)+q_{1} q_{2} \Psi(A, D)+\Psi(B, C)+q_{2} \Psi(B, D)}{\left(1+q_{1}\right)\left(1+q_{2}\right)} .
\end{aligned}
$$

Proof This proof is similar to our proof of Theorem 2.1, so we omit it.

Remark 2.3 From Theorem 2.3, we can deduce the following midpoint special cases. 
Alqudah et al. Advances in Difference Equations

(2021) 2021:264

Page 17 of 29

- If $A=a, B=\frac{q_{1} a+b}{1+q_{1}}, C=\frac{q_{2} d+c}{1+q_{2}}, D=d$, then

$$
\begin{aligned}
& \Psi(\left.\frac{\left(2 q_{1}+q_{1}^{2}\right) a+b}{\left(1+q_{1}\right)^{2}}, \frac{c+\left(2 q_{2}+q_{2}^{2}\right) d}{\left(1+q_{2}\right)^{2}}\right) \\
& \leq \frac{1+q_{1}}{2(b-a)} \int_{a}^{\frac{q_{1} a+b}{1+q_{1}}} \Psi\left(u, \frac{c+\left(2 q_{2}+q_{2}^{2}\right) d}{\left(1+q_{2}\right)^{2}}\right){ }_{a} d_{q_{1}} u \\
&+\frac{1+q_{2}}{2(d-c)} \int_{\frac{q_{2} d+c}{1+q_{2}}}^{d} \Psi\left(\frac{\left(2 q_{1}+q_{1}^{2}\right) a+b}{\left(1+q_{1}\right)^{2}}, v\right){ }^{d} d_{q_{2}} v \\
& \leq \frac{\left(1+q_{1}\right)\left(1+q_{2}\right)}{(b-a)(d-c)} \int_{a}^{\frac{q_{1} a+b}{1+q_{1}}} \int_{\frac{q_{2} d+c}{1+q_{2}}}^{d} \Psi(u, v)^{d} d_{q_{2} v_{a} d_{q_{1}} u} \\
& \leq \frac{1+q_{1}}{2\left(1+q_{2}\right)(b-a)} \int_{a}^{\frac{q_{1} a+b}{1+q_{1}}} \Psi\left(u, \frac{q_{2} d+c}{1+q_{2}}\right){ }_{a} d_{q_{1}} u \\
&+\frac{q_{2}\left(1+q_{1}\right)}{2\left(1+q_{2}\right)(b-a)} \int_{a}^{\frac{q_{1} a+b}{1+q_{1}}} \Psi(u, d)_{a} d_{q_{1}} u \\
&+\frac{q_{1}\left(1+q_{2}\right)}{2\left(1+q_{1}\right)(d-c)} \int_{\frac{q_{2} d+c}{1+q_{2}}}^{d} \Psi(a, v)^{d} d_{q_{2}} v \\
& \quad \frac{q_{1} \Psi\left(a, \frac{q_{2} d+c}{1+q_{2}}\right)+q_{1} q_{2} \Psi(a, d)+\Psi\left(\frac{q_{1} a+b}{1+q_{1}}, \frac{q_{2} d+c}{1+q_{2}}\right)+q_{2} \Psi\left(\frac{q_{1} a+b}{1+q_{1}}, d\right)}{2\left(1+q_{1}\right)(d-c)} \int_{\frac{q_{2} d+c}{1+q_{2}}}^{d} \Psi\left(\frac{q_{1} a+b}{1+q_{1}}, v\right){ }^{d} d_{q_{2}} v \\
& \quad
\end{aligned}
$$

- If $A=a, B=\frac{q_{1} b+a}{1+q_{1}}, C=\frac{q_{2} d+c}{1+q_{2}}, D=d$, then

$$
\begin{aligned}
\Psi( & \left.\frac{\left(1+q_{1}+q_{1}^{2}\right) a+q_{1} b}{\left(1+q_{1}\right)^{2}}, \frac{c+\left(2 q_{2}+q_{2}^{2}\right) d}{\left(1+q_{2}\right)^{2}}\right) \\
\leq & \frac{1+q_{1}}{2 q_{1}(b-a)} \int_{a}^{\frac{q_{1} b+a}{1+q_{1}}} \Psi\left(u, \frac{c+\left(2 q_{2}+q_{2}^{2}\right) d}{\left(1+q_{2}\right)^{2}}\right){ }_{a} d_{q_{1}} u \\
& +\frac{1+q_{2}}{2(d-c)} \int_{\frac{q_{2} d+c}{1+q_{2}}}^{d} \Psi\left(\frac{\left(1+q_{1}+q_{1}^{2}\right) a+q_{1} b}{\left(1+q_{1}\right)^{2}}, v\right)^{d} d_{q_{2}} v \\
\leq & \frac{\left(1+q_{1}\right)\left(1+q_{2}\right)}{q_{1}(b-a)(d-c)} \int_{a}^{\frac{q_{1} b+a}{1+q_{1}}} \int_{\frac{q_{2} d+c}{1+q_{2}}}^{d} \Psi(u, v)^{d} d_{q_{2}} v_{a} d_{q_{1}} u \\
\leq & \frac{1+q_{1}}{2 q_{1}\left(1+q_{2}\right)(b-a)} \int_{a}^{\frac{q_{1} b+a}{1+q_{1}}} \Psi\left(u, \frac{q_{2} d+c}{1+q_{2}}\right){ }_{a} d_{q_{1}} u \\
& +\frac{q_{2}\left(1+q_{1}\right)}{2 q_{1}\left(1+q_{2}\right)(b-a)} \int_{a}^{\frac{q_{1} b+a}{1+q_{1}}} \Psi(u, d)_{a} d_{q_{1}} u \\
& +\frac{q_{1}\left(1+q_{2}\right)}{2\left(1+q_{1}\right)(d-c)} \int_{\frac{q_{2} d+c}{1+q_{2}}}^{d} \Psi(a, v)^{d} d_{q_{2}} v \\
& +\frac{1+q_{2}}{2\left(1+q_{1}\right)(d-c)} \int_{\frac{q_{2} d+c}{1+q_{2}}}^{d} \Psi\left(\frac{q_{1} b+a}{1+q_{1}}, v\right) d d_{q_{2}} v
\end{aligned}
$$




$$
\leq \frac{q_{1} \Psi\left(a, \frac{q_{2} d+c}{1+q_{2}}\right)+q_{1} q_{2} \Psi(a, d)+\Psi\left(\frac{q_{1} b+a}{1+q_{1}}, \frac{q_{2} d+c}{1+q_{2}}\right)+q_{2} \Psi\left(\frac{q_{1} b+a}{1+q_{1}}, d\right)}{\left(1+q_{1}\right)\left(1+q_{2}\right)}
$$

- If $A=a, B=\frac{q_{1} a+b}{1+q_{1}}, C=\frac{q_{2} c+d}{1+q_{2}}, D=d$, then

$$
\begin{aligned}
\Psi( & \left.\frac{\left(2 q_{1}+q_{1}^{2}\right) a+b}{\left(1+q_{1}\right)^{2}}, \frac{q_{2} c+\left(1+q_{2}+q_{2}^{2}\right) d}{\left(1+q_{2}\right)^{2}}\right) \\
\leq & \frac{1+q_{1}}{2(b-a)} \int_{a}^{\frac{q_{1} a+b}{1+q_{1}}} \Psi\left(u, \frac{q_{2} c+\left(1+q_{2}+q_{2}^{2}\right) d}{\left(1+q_{2}\right)^{2}}\right){ }_{a} d_{q_{1}} u \\
& +\frac{1+q_{2}}{2 q_{2}(d-c)} \int_{\frac{q_{2} c+d}{1+q_{2}}}^{d} \Psi\left(\frac{\left(2 q_{1}+q_{1}^{2}\right) a+b}{\left(1+q_{1}\right)^{2}}, v\right){ }^{d} d_{q_{2}} v \\
\leq & \frac{\left(1+q_{1}\right)\left(1+q_{2}\right)}{q_{2}(b-a)(d-c)} \int_{a}^{\frac{q_{1} a+b}{1+q_{1}}} \int_{\frac{q_{2} c+d}{1+q_{2}}}^{d} \Psi(u, v) d d_{q_{2}} v_{a} d_{q_{1}} u \\
\leq & \frac{1+q_{1}}{2\left(1+q_{2}\right)(b-a)} \int_{a}^{\frac{q_{1} a+b}{1+q_{1}}} \Psi\left(u, \frac{q_{2} c+d}{1+q_{2}}\right){ }_{a} d_{q_{1}} u \\
& +\frac{q_{2}\left(1+q_{1}\right)}{2\left(1+q_{2}\right)(b-a)} \int_{a}^{\frac{q_{1} a+b}{1+q_{1}}} \Psi(u, d)_{a} d_{q_{1}} u \\
& +\frac{q_{1}\left(1+q_{2}\right)}{2 q_{2}\left(1+q_{1}\right)(d-c)} \int_{\frac{q_{2} c+d}{1+q_{2}}}^{d} \Psi(a, v)^{d} d_{q_{2}} v \\
& +\frac{1+q_{2}}{2 q_{2}\left(1+q_{1}\right)(d-c)} \int_{\frac{q_{2} c+d}{1+q_{2}}}^{d} \Psi\left(\frac{q_{1} a+b}{1+q_{1}}, v\right){ }^{d} d_{q_{2}} v \\
& \frac{q_{1}\left(a, \frac{q_{2} c+d}{1+q_{2}}\right)+q_{1} q_{2} \Psi(a, d)+\Psi\left(\frac{q_{1} a+b}{1+q_{1}}, \frac{q_{2} c+d}{1+q_{2}}\right)+q_{2} \Psi\left(\frac{q_{1} a+b}{1+q_{1}}, d\right)}{\left(1+q_{1}\right)\left(1+q_{2}\right)}
\end{aligned}
$$

- If $A=a, B=\frac{q_{1} b+a}{1+q_{1}}, C=\frac{q_{2} c+d}{1+q_{2}}, D=d$, then

$$
\begin{aligned}
\Psi( & \left.\frac{\left(1+q_{1}+q_{1}^{2}\right) a+q_{1} b}{\left(1+q_{1}\right)^{2}}, \frac{q_{2} c+\left(1+q_{2}+q_{2}^{2}\right) d}{\left(1+q_{2}\right)^{2}}\right) \\
\leq & \frac{1+q_{1}}{2 q_{1}(b-a)} \int_{a}^{\frac{q_{1} b+a}{1+q_{1}}} \Psi\left(u, \frac{q_{2} c+\left(1+q_{2}+q_{2}^{2}\right) d}{\left(1+q_{2}\right)^{2}}\right){ }_{a} d_{q_{1}} u \\
& +\frac{1+q_{2}}{2 q_{2}(d-c)} \int_{\frac{q_{2} c+d}{1+q_{2}}}^{d} \Psi\left(\frac{\left(1+q_{1}+q_{1}^{2}\right) a+q_{1} b}{\left(1+q_{1}\right)^{2}}, v\right){ }^{d} d_{q_{2}} v \\
\leq & \frac{\left(1+q_{1}\right)\left(1+q_{2}\right)}{q_{1} q_{2}(b-a)(d-c)} \int_{a}^{\frac{q_{1} b+a}{1+q_{1}}} \int_{\frac{q_{2} c+d}{1+q_{2}}}^{d} \Psi(u, v)^{d} d_{q_{2}} v_{a} d_{q_{1}} u \\
\leq & \frac{1+q_{1}}{2 q_{1}\left(1+q_{2}\right)(b-a)} \int_{a}^{\frac{q_{1} b+a}{1+q_{1}}} \Psi\left(u, \frac{q_{2} c+d}{1+q_{2}}\right){ }_{a} d_{q_{1}} u \\
& +\frac{q_{2}\left(1+q_{1}\right)}{2 q_{1}\left(1+q_{2}\right)(b-a)} \int_{a}^{\frac{q_{1} b+a}{1+q_{1}}} \Psi(u, d)_{a} d_{q_{1}} u \\
& +\frac{q_{1}\left(1+q_{2}\right)}{2 q_{2}\left(1+q_{1}\right)(d-c)} \int_{\frac{q_{2} c+d}{1+q_{2}}}^{d} \Psi(a, v)^{d} d_{q_{2}} v
\end{aligned}
$$




$$
\begin{aligned}
& +\frac{1+q_{2}}{2 q_{2}\left(1+q_{1}\right)(d-c)} \int_{\frac{q_{2} c+d}{1+q_{2}}}^{d} \Psi\left(\frac{q_{1} b+a}{1+q_{1}}, v\right) d d_{q_{2}} v \\
\leq & \frac{q_{1} \Psi\left(a, \frac{q_{2} c+d}{1+q_{2}}\right)+q_{1} q_{2} \Psi(a, d)+\Psi\left(\frac{q_{1} b+a}{1+q_{1}}, \frac{q_{2} c+d}{1+q_{2}}\right)+q_{2} \Psi\left(\frac{q_{1} b+a}{1+q_{1}}, d\right)}{\left(1+q_{1}\right)\left(1+q_{2}\right)} .
\end{aligned}
$$

By combining Theorems 1.5, 2.1, 2.2 and Theorem 2.3, we can deduce similar bounds to the inequalities in Theorem 1.1.

Theorem 2.4 Suppose that $\Psi: \Delta \rightarrow \mathbb{R}_{e}$ is a coordinated convex function on $\Delta$ and $\Psi \in$ $L_{1}(\Delta)$. Then we have

$$
\begin{aligned}
2 \Psi( & \left.\frac{A+B}{2}, \frac{C+D}{2}\right) \\
\leq & \frac{1}{2(B-A)}\left[\int_{A}^{B}\left(\Psi\left(u, \frac{C+q_{2} D}{1+q_{2}}\right)+\Psi\left(u, \frac{q_{2} C+D}{1+q_{2}}\right)\right){ }_{A} d_{q_{1}} u\right. \\
& \left.+\int_{A}^{B}\left(\Psi\left(u, \frac{C+q_{2} D}{1+q_{2}}\right)+\Psi\left(u, \frac{q_{2} C+D}{1+q_{2}}\right)\right)^{B} d_{q_{1}} u\right] \\
& +\frac{1}{2(D-C)}\left[\int_{C}^{D}\left(\Psi\left(\frac{A+q_{1} B}{1+q_{1}}, v\right)+\Psi\left(\frac{q_{1} A+B}{1+q_{1}}, v\right)\right)_{C} d_{q_{2}} v\right. \\
& \left.+\int_{C}^{D}\left(\Psi\left(\frac{A+q_{1} B}{1+q_{1}}, v\right)+\Psi\left(\frac{q_{1} A+B}{1+q_{1}}, v\right)\right){ }^{D} d_{q_{2}} v\right] \\
\leq & \frac{1}{(B-A)(D-C)}\left[\int_{A}^{B} \int_{C}^{D} \Psi(u, v){ }^{D} d_{q_{2}} v_{A} d_{q_{1}} u+\int_{A}^{B} \int_{C}^{D} \Psi(u, v)^{D} d_{q_{2}} v^{B} d_{q_{1}} u\right. \\
& \left.+\int_{A}^{B} \int_{C}^{D} \Psi(u, v) C d_{q_{2}} v^{B} d_{q_{1}} u+\int_{A}^{B} \int_{C}^{D} \Psi(u, v)_{C} d_{q_{2}} v_{A} d_{q_{1}} u\right] \\
\leq & \frac{1}{2(B-A)} \int_{A}^{B}(\Psi(u, C)+\Psi(u, D))_{A} d_{q_{1}} u \\
& +\frac{1}{2(B-A)} \int_{A}^{B}(\Psi(u, C)+\Psi(u, D))^{B} d_{q_{1}} u \\
& +\frac{1}{2(D-C)} \int_{A}^{B}(\Psi(A, v)+\Psi(B, v)){ }_{C} d_{q_{2}} v \\
& \left.+\frac{1}{2(D-C)} \int_{A}^{B}(\Psi(A, v)+\Psi(B, v))^{D} d_{q_{2}} v\right] \\
\leq & \Psi(A, C)+\Psi(A, D)+\Psi(B, C)+\Psi(B, D) .
\end{aligned}
$$

Proof It suffices to see that we have, due to the coordinated convexity of $\Psi$,

$$
\begin{aligned}
4 \Psi\left(\frac{A+B}{2}, \frac{C+D}{2}\right)= & 4 \Psi\left(\frac{q_{1} A+B+A+q_{1} B}{2\left(1+q_{1}\right)}, \frac{q_{2} C+D+C+q_{2} D}{2\left(1+q_{2}\right)}\right) \\
\leq & \Psi\left(\frac{q_{1} A+B}{1+q_{1}}, \frac{q_{2} C+D}{1+q_{2}}\right)+\Psi\left(\frac{q_{1} A+B}{1+q_{1}}, \frac{C+q_{2} D}{1+q_{2}}\right) \\
& +\Psi\left(\frac{A+q_{1} B}{1+q_{1}}, \frac{q_{2} C+D}{1+q_{2}}\right)+\Psi\left(\frac{A+q_{1} B}{1+q_{1}}, \frac{C+q_{2} D}{1+q_{2}}\right) .
\end{aligned}
$$


Then, we can obtain the desired inequality by utilizing Theorems 1.5, 2.1, 2.2 and Theorem 2.3 .

Remark 2.4 If $q_{1}, q_{2} \rightarrow 1^{-}$in Theorem 2.4, then Theorem 2.4 reduces to Theorem 1.1.

Finally, by utilizing Remarks 1.1, 2.1, 2.2 and Remark 2.3, we obtain the following refinements for previous results.

Theorem 2.5 Let $\Psi: \Delta \rightarrow \mathbb{R}_{e}$ be a coordinated convex function on $\Delta$ and $q_{1}, q_{2} \in(0,1)$, then we have

$$
\begin{aligned}
& 4 \Psi\left(\frac{a+b}{2}, \frac{c+d}{2}\right) \\
& \leq \Psi\left(\frac{\left(2 q_{1}+q_{1}^{2}\right) a+b}{\left(1+q_{1}\right)^{2}}, \frac{\left(2 q_{2}+q_{2}^{2}\right) c+d}{\left(1+q_{2}\right)^{2}}\right)+\Psi\left(\frac{\left(2 q_{1}+q_{1}^{2}\right) a+b}{\left(1+q_{1}\right)^{2}}, \frac{\left(2 q_{2}+q_{2}^{2}\right) d+c}{\left(1+q_{2}\right)^{2}}\right) \\
& +\Psi\left(\frac{\left(2 q_{1}+q_{1}^{2}\right) b+a}{\left(1+q_{1}\right)^{2}}, \frac{\left(2 q_{2}+q_{2}^{2}\right) c+d}{\left(1+q_{2}\right)^{2}}\right)+\Psi\left(\frac{\left(2 q_{1}+q_{1}^{2}\right) b+a}{\left(1+q_{1}\right)^{2}}, \frac{\left(2 q_{2}+q_{2}^{2}\right) d+c}{\left(1+q_{2}\right)^{2}}\right) \\
& \leq \frac{1+q_{1}}{2(b-a)}\left[\int_{a}^{\frac{q_{1} a+b}{1+q_{1}}} \Psi\left(u, \frac{\left(2 q_{2}+q_{2}^{2}\right) c+d}{\left(1+q_{2}\right)^{2}}\right){ }_{a} d_{q_{1}} u\right. \\
& +\int_{\frac{q_{1} b+a}{1+q_{1}}}^{b} \Psi\left(u, \frac{c+\left(2 q_{2}+q_{2}^{2}\right) d}{\left(1+q_{2}\right)^{2}}\right) b d_{q_{1}} u \\
& \left.+\int_{\frac{q_{1} b+a}{1+q_{1}}}^{b} \Psi\left(u, \frac{\left(2 q_{2}+q_{2}^{2}\right) c+d}{\left(1+q_{2}\right)^{2}}\right){ }^{b} d_{q_{1}} u+\int_{a}^{\frac{q_{1} a+b}{1+q_{1}}} \Psi\left(u, \frac{c+\left(2 q_{2}+q_{2}^{2}\right) d}{\left(1+q_{2}\right)^{2}}\right){ }_{a} d_{q_{1}} u\right] \\
& +\frac{1+q_{2}}{2(d-c)}\left[\int_{c}^{\frac{q_{2} c+d}{1+q_{2}}} \Psi\left(\frac{\left(2 q_{1}+q_{1}^{2}\right) a+b}{\left(1+q_{1}\right)^{2}}, v\right){ }_{c} d_{q_{2}} v\right. \\
& +\int_{\frac{q_{2} d+c}{1+q_{2}}}^{d} \Psi\left(\frac{a+\left(2 q_{1}+q_{1}^{2}\right) b}{\left(1+q_{1}\right)^{2}}, v\right) d d_{q_{2}} v \\
& \left.+\int_{c}^{\frac{q_{2} c+d}{1+q_{2}}} \Psi\left(\frac{a+\left(2 q_{1}+q_{1}^{2}\right) b}{\left(1+q_{1}\right)^{2}}, v\right){ }_{c} d_{q_{2}} v+\int_{\frac{q_{2} d+c}{1+q_{2}}}^{d} \Psi\left(\frac{\left(2 q_{1}+q_{1}^{2}\right) a+b}{\left(1+q_{1}\right)^{2}}, v\right){ }^{d} d_{q_{2}} v\right] \\
& \leq \frac{\left(1+q_{1}\right)\left(1+q_{2}\right)}{(b-a)(d-c)}\left[\int_{a}^{\frac{q_{1} a+b}{1+q_{1}}} \int_{c}^{\frac{q_{2} c+d}{1+q_{2}}} \Psi(u, v){ }_{c} d_{q_{2}} v_{a} d_{q_{1}} u\right. \\
& +\int_{\frac{q_{1} b+a}{1+q_{1}}}^{b} \int_{\frac{q_{2} d+c}{1+q_{2}}}^{d} \Psi(u, v)^{d} d_{q_{2}} v^{b} d_{q_{1}} u \\
& \left.+\int_{\frac{q_{1} b+a}{1+q_{1}}}^{b} \int_{c}^{\frac{q_{2} c+d}{1+q_{2}}} \Psi(u, v){ }_{c} d_{q_{2}} v^{b} d_{q_{1}} u+\int_{a}^{\frac{q_{1} a+b}{1+q_{1}}} \int_{\frac{q_{2} d+c}{1+q_{2}}}^{d} \Psi(u, v)^{d} d_{q_{2}} v_{a} d_{q_{1}} u\right] \\
& \leq \frac{1+q_{1}}{2\left(1+q_{2}\right)(b-a)}\left[q_{2} \int_{a}^{\frac{q_{1} a+b}{1+q_{1}}} \Psi(u, c)_{a} d_{q_{1}} u+\int_{a}^{\frac{q_{1} a+b}{1+q_{1}}} \Psi\left(u, \frac{q_{2} c+d}{1+q_{2}}\right){ }_{a} d_{q_{1}} u\right. \\
& +\int_{\frac{q_{1} b+a}{1+q_{1}}}^{b} \Psi\left(u, \frac{q_{2} d+c}{1+q_{2}}\right){ }^{b} d_{q_{1}} u+q_{2} \int_{\frac{q_{1} b+a}{1+q_{1}}}^{b} \Psi(u, d)^{b} d_{q_{1}} u
\end{aligned}
$$




$$
\begin{aligned}
& +q_{2} \int_{\frac{q_{1} b+a}{1+q_{1}}}^{b} \Psi(u, c)^{b} d_{q_{1}} u+\int_{\frac{q_{1} b+a}{1+q_{1}}}^{b} \Psi\left(u, \frac{q_{2} c+d}{1+q_{2}}\right){ }^{b} d_{q_{1}} u \\
& \left.+q_{2} \int_{a}^{\frac{q_{1} a+b}{1+q_{1}}} \Psi(u, d){ }_{a} d_{q_{1}} u+\int_{a}^{\frac{q_{1} a+b}{1+q_{1}}} \Psi\left(u, \frac{q_{2} d+c}{1+q_{2}}\right){ }_{a} d_{q_{1}} u\right] \\
& +\frac{1+q_{2}}{2\left(1+q_{1}\right)(d-c)}\left[q_{1} \int_{c}^{\frac{q_{2} c+d}{1+q_{2}}} \Psi(a, v){ }_{c} d_{q_{2}} v\right. \\
& +\int_{c}^{\frac{q_{2} c+d}{1+q_{2}}} \Psi\left(\frac{q_{1} a+b}{1+q_{1}}, v\right){ }_{c} d_{q_{2}} v+\int_{\frac{q_{2} d+c}{1+q_{2}}}^{d} \Psi\left(\frac{q_{1} b+a}{1+q_{1}}, v\right) d d_{q_{2}} v \\
& +q_{1} \int_{\frac{q_{2} d+c}{1+q_{2}}}^{d} \Psi(b, v)^{d} d_{q_{2}} v+\int_{c}^{\frac{q_{2} c+d}{1+q_{2}}} \Psi\left(\frac{q_{1} b+a}{1+q_{1}}, v\right){ }_{c} d_{q_{2}} v \\
& +q_{1} \int_{c}^{\frac{q_{2} c+d}{1+q_{2}}} \Psi(b, v)_{c} d_{q_{2}} v+\int_{\frac{q_{2} d+c}{1+q_{2}}}^{d} \Psi\left(\frac{q_{1} a+b}{1+q_{1}}, v\right) d d_{q_{2}} v \\
& \left.+q_{1} \int_{\frac{q_{2} d+c}{1+q_{2}}}^{d} \Psi(a, v)^{d} d_{q_{2}} v\right] \\
& \leq \frac{q_{1} q_{2}[\Psi(a, c)+\Psi(a, d)+\Psi(b, d)+\Psi(b, c)]}{\left(1+q_{1}\right)\left(1+q_{2}\right)} \\
& +\frac{q_{1}\left[\Psi\left(a, \frac{q_{2} c+d}{1+q_{2}}\right)+\Psi\left(a, \frac{q_{2} d+c}{1+q_{2}}\right)+\Psi\left(b, \frac{q_{2} c+d}{1+q_{2}}\right)+\Psi\left(b, \frac{q_{2} d+c}{1+q_{2}}\right)\right]}{\left(1+q_{1}\right)\left(1+q_{2}\right)} \\
& +\frac{q_{2}\left[\Psi\left(\frac{q_{1} a+b}{1+q_{1}}, c\right)+\Psi\left(\frac{q_{1} b+a}{1+q_{1}}, d\right)+\Psi\left(\frac{q_{1} b+a}{1+q_{1}}, c\right)+\Psi\left(\frac{q_{1} a+b}{1+q_{1}}, d\right)\right]}{\left(1+q_{1}\right)\left(1+q_{2}\right)} \\
& +\frac{\Psi\left(\frac{q_{1} a+b}{1+q_{1}}, \frac{q_{2} c+d}{1+q_{2}}\right)+\Psi\left(\frac{q_{1} a+b}{1+q_{1}}, \frac{q_{2} d+c}{1+q_{2}}\right)+\Psi\left(\frac{q_{1} b+a}{1+q_{1}}, \frac{q_{2} c+d}{1+q_{2}}\right)+\Psi\left(\frac{q_{1} b+a}{1+q_{1}}, \frac{q_{2} d+c}{1+q_{2}}\right)}{\left(1+q_{1}\right)\left(1+q_{2}\right)} \\
& \leq \Psi(a, c)+\Psi(a, d)+\Psi(b, c)+\Psi(b, d) .
\end{aligned}
$$

Proof By summing up the inequalities (1.30), (2.12), (2.19) and (2.21), we can obtain the second, third, fourth and fifth inequalities of the desired inequality (2.26). The last inequality is the consequence of the coordinated convexity of the function $\Psi$.

For the first inequality, we note that

$$
\begin{aligned}
4 \Psi( & \left.\frac{a+b}{2}, \frac{c+d}{2}\right) \\
= & 4 \Psi\left(\frac{\left(2 q_{1}+q_{1}^{2}\right) a+b+a+\left(2 q_{1}+q_{1}^{2}\right) b}{2\left(1+q_{1}^{2}\right)}, \frac{\left(2 q_{2}+q_{2}^{2}\right) c+d+c+\left(2 q_{2}+q_{2}^{2}\right) d}{2\left(1+q_{1}^{2}\right)}\right) \\
\leq & \Psi\left(\frac{\left(2 q_{1}+q_{1}^{2}\right) a+b}{\left(1+q_{1}\right)^{2}}, \frac{\left(2 q_{2}+q_{2}^{2}\right) c+d}{\left(1+q_{2}\right)^{2}}\right)+\Psi\left(\frac{\left(2 q_{1}+q_{1}^{2}\right) a+b}{\left(1+q_{1}\right)^{2}}, \frac{\left(2 q_{2}+q_{2}^{2}\right) d+c}{\left(1+q_{2}\right)^{2}}\right) \\
& +\Psi\left(\frac{\left(2 q_{1}+q_{1}^{2}\right) b+a}{\left(1+q_{1}\right)^{2}}, \frac{\left(2 q_{2}+q_{2}^{2}\right) c+d}{\left(1+q_{2}\right)^{2}}\right) \\
& +\Psi\left(\frac{\left(2 q_{1}+q_{1}^{2}\right) b+a}{\left(1+q_{1}\right)^{2}}, \frac{\left(2 q_{2}+q_{2}^{2}\right) d+c}{\left(1+q_{2}\right)^{2}}\right),
\end{aligned}
$$

which completes our proof. 
In a similar way, by using inequalities (1.29), (2.13), (2.16) and (2.24), we can deduce the following theorem.

Theorem 2.6 Let $\Psi: \Delta \rightarrow \mathbb{R}_{e}$ be a coordinated convex function on $\Delta$ and $q_{1}, q_{2} \in(0,1)$, then we have

$$
\begin{aligned}
& 4 \Psi\left(\frac{a+b}{2}, \frac{c+d}{2}\right) \\
& \leq \Psi\left(\frac{\left(1+q_{1}+q_{1}^{2}\right) a+q_{1} b}{\left(1+q_{1}\right)^{2}}, \frac{\left(1+q_{2}+q_{2}^{2}\right) c+q_{2} d}{\left(1+q_{2}\right)^{2}}\right) \\
& +\Psi\left(\frac{\left(1+q_{1}+q_{1}^{2}\right) a+q_{1} b}{\left(1+q_{1}\right)^{2}}, \frac{q_{2} c+\left(1+q_{2}+q_{2}^{2}\right) d}{\left(1+q_{2}\right)^{2}}\right) \\
& +\Psi\left(\frac{q_{1} a+\left(1+q_{1}+q_{1}^{2}\right) b}{\left(1+q_{1}\right)^{2}}, \frac{\left(1+q_{2}+q_{2}^{2}\right) c+q_{2} d}{\left(1+q_{2}\right)^{2}}\right) \\
& +\Psi\left(\frac{q_{1} a+\left(1+q_{1}+q_{1}^{2}\right) b}{\left(1+q_{1}\right)^{2}}, \frac{q_{2} c+\left(1+q_{2}+q_{2}^{2}\right) d}{\left(1+q_{2}\right)^{2}}\right) \\
& \leq \frac{1+q_{1}}{2 q_{1}(b-a)}\left[\int_{a}^{\frac{q_{1} b+a}{1+q_{1}}} \Psi\left(u, \frac{\left(1+q_{2}+q_{2}^{2}\right) c+q_{2} d}{\left(1+q_{2}\right)^{2}}\right){ }_{a} d_{q_{1}} u\right. \\
& +\int_{\frac{q_{1} a+b}{1+q_{1}}}^{b} \Psi\left(u, \frac{q_{2} c+\left(1+q_{2}+q_{2}^{2}\right) d}{\left(1+q_{2}\right)^{2}}\right) b d_{q_{1}} u \\
& +\int_{\frac{q_{1} a+b}{1+q_{1}}}^{b} \Psi\left(u, \frac{\left(1+q_{2}+q_{2}^{2}\right) c+q_{2} d}{\left(1+q_{2}\right)^{2}}\right) b d_{q_{1}} u \\
& \left.+\int_{a}^{\frac{q_{1} b+a}{1+q_{1}}} \Psi\left(u, \frac{q_{2} c+\left(1+q_{2}+q_{2}^{2}\right) d}{\left(1+q_{2}\right)^{2}}\right){ }_{a} d_{q_{1}} u\right] \\
& +\frac{1+q_{2}}{2 q_{2}(d-c)}\left[\int_{c}^{\frac{q_{2} d+c}{1+q_{2}}} \Psi\left(\frac{\left(1+q_{1}+q_{1}^{2}\right) a+q_{1} b}{\left(1+q_{1}\right)^{2}}, v\right){ }_{c} d_{q_{2}} v\right. \\
& +\int_{\frac{q_{2} c+d}{1+q_{2}}}^{d} \Psi\left(\frac{q_{1} a+\left(1+q_{1}+q_{1}^{2}\right) b}{\left(1+q_{1}\right)^{2}}, v\right)^{d} d_{q_{2}} v \\
& +\int_{c}^{\frac{q_{2} d+c}{1+q_{2}}} \Psi\left(\frac{q_{1} a+\left(1+q_{1}+q_{1}^{2}\right) b}{\left(1+q_{1}\right)^{2}}, v\right){ }_{c} d_{q_{2}} v \\
& \left.+\int_{\frac{q_{2} c+d}{1+q_{2}}}^{d} \Psi\left(\frac{\left(1+q_{1}+q_{1}^{2}\right) a+q_{1} b}{\left(1+q_{1}\right)^{2}}, v\right) d d_{q_{2}} v\right] \\
& \leq \frac{\left(1+q_{1}\right)\left(1+q_{2}\right)}{q_{1} q_{2}(b-a)(d-c)}\left[\int_{a}^{\frac{q_{1} b+a}{1+q_{1}}} \int_{c}^{\frac{q_{2} d+c}{1+q_{2}}} \Psi(u, v){ }_{c} d_{q_{2}} v_{a} d_{q_{1}} u\right. \\
& +\int_{\frac{q_{1} a+b}{1+q_{1}}}^{b} \int_{\frac{q_{2} c+d}{1+q_{2}}}^{d} \Psi(u, v)^{d} d_{q_{2}} v^{b} d_{q_{1}} u \\
& \left.+\int_{\frac{q_{1} a+b}{1+q_{1}}}^{b} \int_{c}^{\frac{q_{2} d+c}{1+q_{2}}} \Psi(u, v)_{c} d_{q_{2}} v^{b} d_{q_{1}} u+\int_{a}^{\frac{q_{1} b+a}{1+q_{1}}} \int_{\frac{q_{2} c+d}{1+q_{2}}}^{d} \Psi(u, v)^{d} d_{q_{2}} v_{a} d_{q_{1}} u\right] \\
& \leq \frac{1+q_{1}}{2 q_{1}\left(1+q_{2}\right)(b-a)}\left[q_{2} \int_{a}^{\frac{q_{1} b+a}{1+q_{1}}} \Psi(u, c)_{a} d_{q_{1}} u\right.
\end{aligned}
$$




$$
\begin{aligned}
& +\int_{a}^{\frac{q_{1} b+a}{1+q_{1}}} \Psi\left(u, \frac{q_{2} d+c}{1+q_{2}}\right){ }_{a} d_{q_{1}} u \\
& +\int_{\frac{q_{1} a+b}{1+q_{1}}}^{b} \Psi\left(u, \frac{q_{2} c+d}{1+q_{2}}\right){ }^{b} d_{q_{1}} u+q_{2} \int_{\frac{q_{1} a+b}{1+q_{1}}}^{b} \Psi(u, d)^{b} d_{q_{1}} u \\
& +q_{2} \int_{\frac{q_{1} a+b}{1+q_{1}}}^{b} \Psi(u, c)^{b} d_{q_{1}} u+\int_{\frac{q_{1} a+b}{1+q_{1}}}^{b} \Psi\left(u, \frac{q_{2} d+c}{1+q_{2}}\right){ }^{b} d_{q_{1}} u \\
& \left.+q_{2} \int_{a}^{\frac{q_{1} b+a}{1+q_{1}}} \Psi(u, d){ }_{a} d_{q_{1}} u+\int_{a}^{\frac{q_{1} b+a}{1+q_{1}}} \Psi\left(u, \frac{q_{2} c+d}{1+q_{2}}\right){ }_{a} d_{q_{1}} u\right] \\
& +\frac{1+q_{2}}{2 q_{2}\left(1+q_{1}\right)(d-c)}\left[q_{1} \int_{c}^{\frac{q_{2} d+c}{1+q_{2}}} \Psi(a, v){ }_{c} d_{q_{2}} v\right. \\
& +\int_{c}^{\frac{q_{2} d+c}{1+q_{2}}} \Psi\left(\frac{q_{1} b+a}{1+q_{1}}, v\right){ }_{c} d_{q_{2}} v \\
& +\int_{\frac{q_{2} c+d}{1+q_{2}}}^{d} \Psi\left(\frac{q_{1} a+b}{1+q_{1}}, v\right) d^{d} d_{q_{2}} v+q_{1} \int_{\frac{q_{2} c+d}{1+q_{2}}}^{d} \Psi(b, v){ }^{d} d_{q_{2}} v \\
& +\int_{c}^{\frac{q_{2} d+c}{1+q_{2}}} \Psi\left(\frac{q_{1} a+b}{1+q_{1}}, v\right){ }_{c} d_{q_{2}} v+q_{1} \int_{c}^{\frac{q_{2} d+c}{1+q_{2}}} \Psi(b, v)_{c} d_{q_{2}} v \\
& \left.+\int_{\frac{q_{2} c+d}{1+q_{2}}}^{d} \Psi\left(\frac{q_{1} b+a}{1+q_{1}}, v\right) d d_{q_{2}} v+q_{1} \int_{\frac{q_{2} c+d}{1+q_{2}}}^{d} \Psi(a, v)^{d} d_{q_{2}} v\right] \\
& \leq \frac{q_{1} q_{2}[\Psi(a, c)+\Psi(a, d)+\Psi(b, d)+\Psi(b, c)]}{\left(1+q_{1}\right)\left(1+q_{2}\right)} \\
& +\frac{q_{1}\left[\Psi\left(a, \frac{q_{2} d+c}{1+q_{2}}\right)+\Psi\left(a, \frac{q_{2} c+d}{1+q_{2}}\right)+\Psi\left(b, \frac{q_{2} c+d}{1+q_{2}}\right)+\Psi\left(b, \frac{q_{2} d+c}{1+q_{2}}\right)\right]}{\left(1+q_{1}\right)\left(1+q_{2}\right)} \\
& +\frac{q_{2}\left[\Psi\left(\frac{q_{1} a+b}{1+q_{1}}, c\right)+\Psi\left(\frac{q_{1} b+a}{1+q_{1}}, d\right)+\Psi\left(\frac{q_{1} b+a}{1+q_{1}}, c\right)+\Psi\left(\frac{q_{1} a+b}{1+q_{1}}, d\right)\right]}{\left(1+q_{1}\right)\left(1+q_{2}\right)} \\
& +\frac{\Psi\left(\frac{q_{1} a+b}{1+q_{1}}, \frac{q_{2} c+d}{1+q_{2}}\right)+\Psi\left(\frac{q_{1} a+b}{1+q_{1}}, \frac{q_{2} d+c}{1+q_{2}}\right)+\Psi\left(\frac{q_{1} b+a}{1+q_{1}}, \frac{q_{2} c+d}{1+q_{2}}\right)+\Psi\left(\frac{q_{1} b+a}{1+q_{1}}, \frac{q_{2} d+c}{1+q_{2}}\right)}{\left(1+q_{1}\right)\left(1+q_{2}\right)} \\
& \leq \Psi(a, c)+\Psi(a, d)+\Psi(b, c)+\Psi(b, d) .
\end{aligned}
$$

In a similar way, by using inequalities (1.31), (2.11), (2.18) and (2.22), we can deduce the following theorem.

Theorem 2.7 Let $\Psi: \Delta \rightarrow \mathbb{R}_{e}$ be $\Delta$ a coordinated convex function on $\Delta$ and $q_{1}, q_{2} \in(0,1)$, then one has

$$
\begin{aligned}
4 \Psi\left(\frac{a+b}{2}, \frac{c+d}{2}\right) \\
\leq \Psi\left(\frac{\left(1+q_{1}+q_{1}^{2}\right) a+q_{1} b}{\left(1+q_{1}\right)^{2}}, \frac{\left(2 q_{2}+q_{2}^{2}\right) c+d}{\left(1+q_{2}\right)^{2}}\right) \\
\quad+\Psi\left(\frac{q_{1} a+\left(1+q_{1}+q_{1}^{2}\right) b}{\left(1+q_{1}\right)^{2}}, \frac{c+\left(2 q_{2}+q_{2}^{2}\right) d}{\left(1+q_{2}\right)^{2}}\right)
\end{aligned}
$$


Alqudah et al. Advances in Difference Equations

(2021) 2021:264

Page 24 of 29

$$
\begin{aligned}
& +\Psi\left(\frac{q_{1} a+\left(1+q_{1}+q_{1}^{2}\right) b}{\left(1+q_{1}\right)^{2}}, \frac{c\left(2 q_{2}+q_{2}^{2}\right)+d}{\left(1+q_{2}\right)^{2}}\right) \\
& +\Psi\left(\frac{\left(1+q_{1}+q_{1}^{2}\right) a+q_{1} b}{\left(1+q_{1}\right)^{2}}, \frac{c+\left(2 q_{2}+q_{2}^{2}\right) d}{\left(1+q_{2}\right)^{2}}\right) \\
& \leq \frac{1+q_{1}}{2 q_{1}(b-a)}\left[\int_{a}^{\frac{q_{1} b+a}{1+q_{1}}} \Psi\left(u, \frac{\left(2 q_{2}+q_{2}^{2}\right) c+d}{\left(1+q_{2}\right)^{2}}\right){ }_{a} d_{q_{1}} u\right. \\
& +\int_{\frac{q_{1} a+b}{1+q_{1}}}^{b} \Psi\left(u, \frac{c+\left(2 q_{2}+q_{2}^{2}\right) d}{\left(1+q_{2}\right)^{2}}\right){ }^{b} d_{q_{1}} u \\
& \left.+\int_{\frac{q_{1} a+b}{1+q_{1}}}^{b} \Psi\left(u, \frac{c\left(2 q_{2}+q_{2}^{2}\right)+d}{\left(1+q_{2}\right)^{2}}\right){ }^{b} d_{q_{1}} u+\int_{a}^{\frac{q_{1} b+a}{1+q_{1}}} \Psi\left(u, \frac{c+\left(2 q_{2}+q_{2}^{2}\right) d}{\left(1+q_{2}\right)^{2}}\right){ }_{a} d_{q_{1}} u\right] \\
& +\frac{1+q_{2}}{2(d-c)}\left[\int_{c}^{\frac{q_{2} c+d}{1+q_{2}}} \Psi\left(\frac{\left(1+q_{1}+q_{1}^{2}\right) a+q_{1} b}{\left(1+q_{1}\right)^{2}}, v\right){ }_{c} d_{q_{2}} v\right. \\
& +\int_{\frac{q_{2} d+c}{1+q_{2}}}^{d} \Psi\left(\frac{q_{1} a+\left(1+q_{1}+q_{1}^{2}\right) b}{\left(1+q_{1}\right)^{2}}, v\right) d d_{q_{2}} v \\
& +\int_{c}^{\frac{q_{2} c+d}{1+q_{2}}} \Psi\left(\frac{q_{1} a+\left(1+q_{1}+q_{1}^{2}\right) b}{\left(1+q_{1}\right)^{2}}, v\right){ }_{c} d_{q_{2}} v \\
& \left.+\int_{\frac{q_{2} d+c}{1+q_{2}}}^{d} \Psi\left(\frac{\left(1+q_{1}+q_{1}^{2}\right) a+q_{1} b}{\left(1+q_{1}\right)^{2}}, v\right) d d_{q_{2}} v\right] \\
& \leq \frac{\left(1+q_{1}\right)\left(1+q_{2}\right)}{q_{1}(b-a)(d-c)}\left[\int_{a}^{\frac{q_{1} b+a}{1+q_{1}}} \int_{c}^{\frac{q_{2} c+d}{1+q_{2}}} \Psi(u, v){ }_{c} d_{q_{2}} v_{a} d_{q_{1}} u\right. \\
& +\int_{\frac{q_{1} a+b}{1+q_{1}}}^{b} \int_{\frac{q_{2} d+c}{1+q_{2}}}^{d} \Psi(u, v)^{d} d_{q_{2}} v^{b} d_{q_{1}} u \\
& \left.+\int_{\frac{q_{1} a+b}{1+q_{1}}}^{b} \int_{c}^{\frac{q_{2} c+d}{1+q_{2}}} \Psi(u, v)_{c} d_{q_{2}} v^{b} d_{q_{1}} u+\int_{a}^{\frac{q_{1} b+a}{1+q_{1}}} \int_{\frac{q_{2} d+c}{1+q_{2}}}^{d} \Psi(u, v)^{d} d_{q_{2}} v_{a} d_{q_{1}} u\right] \\
& \leq \frac{\left(1+q_{1}\right)}{2 q_{1}\left(1+q_{2}\right)(b-a)}\left[q_{2} \int_{a}^{\frac{q_{1} b+a}{1+q_{1}}} \Psi(u, c)_{a} d_{q_{1}} u+\int_{a}^{\frac{q_{1} b+a}{1+q_{1}}} \Psi\left(u, \frac{q_{2} d+c}{1+q_{2}}\right){ }_{a} d_{q_{1}} u\right. \\
& +\int_{\frac{q_{1} a+b}{1+q_{1}}}^{b} \Psi\left(u, \frac{q_{2} d+c}{1+q_{2}}\right){ }^{b} d_{q_{1}} u+q_{2} \int_{\frac{q_{1} a+b}{1+q_{1}}}^{b} \Psi(u, d)^{b} d_{q_{1}} u \\
& +q_{2} \int_{\frac{q_{1} a+b}{1+q_{1}}}^{b} \Psi(u, c)^{b} d_{q_{1}} u+\int_{\frac{q_{1} a+b}{1+q_{1}}}^{b} \Psi\left(u, \frac{q_{2} d+c}{1+q_{2}}\right){ }^{b} d_{q_{1}} u \\
& \left.+\int_{a}^{\frac{q_{1} b+a}{1+q_{1}}} \Psi\left(u, \frac{q_{2} d+c}{1+q_{2}}\right){ }_{a} d_{q_{1}} u+q_{2} \int_{a}^{\frac{q_{1} b+a}{1+q_{1}}} \Psi(u, d)_{a} d_{q_{1}} u\right] \\
& +\frac{\left(1+q_{2}\right)}{2\left(1+q_{1}\right)(d-c)}\left[q_{1} \int_{c}^{\frac{q_{2} c+d}{1+q_{2}}} \Psi(a, v){ }_{c} d_{q_{2}} v+\int_{c}^{\frac{q_{2} c+d}{1+q_{2}}} \Psi\left(\frac{q_{1} b+a}{1+q_{1}}, v\right){ }_{c} d_{q_{2}} v\right. \\
& +\int_{\frac{q_{2} d+c}{1+q_{2}}}^{d} \Psi\left(\frac{q_{1} a+b}{1+q_{1}}, v\right) d d_{q_{2}} v+q_{1} \int_{\frac{q_{2} d+c}{1+q_{2}}}^{d} \Psi(b, v)^{d} d_{q_{2}} v \\
& +\int_{c}^{\frac{q_{2} c+d}{1+q_{2}}} \Psi\left(\frac{q_{1} a+b}{1+q_{1}}, v\right){ }_{c} d_{q_{2}} v+q_{1} \int_{c}^{\frac{q_{2} c+d}{1+q_{2}}} \Psi(b, v){ }_{c} d_{q_{2}} v
\end{aligned}
$$




$$
\begin{aligned}
+ & \left.q_{1} \int_{\frac{q_{2} d+c}{1+q_{2}}}^{d} \Psi(a, v){ }^{d} d_{q_{2}} v+\int_{\frac{q_{2} d+c}{1+q_{2}}}^{d} \Psi\left(\frac{q_{1} b+a}{1+q_{1}}, v\right){ }^{d} d_{q_{2}} v\right] \\
\leq & \frac{q_{1} q_{2}[\Psi(a, c)+\Psi(a, d)+\Psi(b, c)+\Psi(b, d)]}{\left(1+q_{1}\right)\left(1+q_{2}\right)} \\
& +\frac{q_{1}\left[\Psi\left(a, \frac{q_{2} c+d}{1+q_{2}}\right)+\Psi\left(b, \frac{q_{2} c+d}{1+q_{2}}\right)+\Psi\left(a, \frac{q_{2} d+c}{1+q_{2}}\right)+\Psi\left(b, \frac{q_{2} d+c}{1+q_{2}}\right)\right]}{\left(1+q_{1}\right)\left(1+q_{2}\right)} \\
& +\frac{q_{2}\left[\Psi\left(\frac{q_{1} a+b}{1+q_{1}}, c\right)+\Psi\left(\frac{q_{1} b+a}{1+q_{1}}, d\right)+\Psi\left(\frac{q_{1} b+a}{1+q_{1}}, c\right)+\Psi\left(\frac{q_{1} a+b}{1+q_{1}}, d\right)\right]}{\left(1+q_{1}\right)\left(1+q_{2}\right)} \\
& +\frac{\Psi\left(\frac{q_{1} a+b}{1+q_{1}}, \frac{q_{2} d+c}{1+q_{2}}\right)+\Psi\left(\frac{q_{1} a+b}{1+q_{1}}, \frac{q_{2} c+d}{1+q_{2}}\right)+\Psi\left(\frac{q_{1} b+a}{1+q_{1}}, \frac{q_{2} c+d}{1+q_{2}}\right)+\Psi\left(\frac{q_{1} b+a}{1+q_{1}}, \frac{q_{2} d+c}{1+q_{2}}\right)}{\left(1+q_{1}\right)\left(1+q_{2}\right)} \\
\leq & \Psi(a, c)+\Psi(a, d)+\Psi(b, c)+\Psi(b, d) .
\end{aligned}
$$

Also, by using inequalities (1.28), (2.14), (2.17) and (2.23), we can deduce the following theorem.

Theorem 2.8 Let $\Psi: \Delta \rightarrow \mathbb{R}_{e}$ be a coordinated convex function on $\Delta$ and $q_{1}, q_{2} \in(0,1)$, then one has

$$
\begin{aligned}
4 \Psi( & \left.\frac{a+b}{2}, \frac{c+d}{2}\right) \\
\leq & \Psi\left(\frac{\left(2 q_{1}+q_{1}^{2}\right) a+b}{\left(1+q_{1}\right)^{2}}, \frac{\left(1+q_{2}+q_{2}^{2}\right) c+q_{2} d}{\left(1+q_{2}\right)^{2}}\right) \\
& +\Psi\left(\frac{a+\left(2 q_{1}+q_{1}^{2}\right) b}{\left(1+q_{1}\right)^{2}}, \frac{q_{2} c+\left(1+q_{2}+q_{2}^{2}\right) d}{\left(1+q_{2}\right)^{2}}\right) \\
& +\Psi\left(\frac{a+\left(2 q_{1}+q_{1}^{2}\right) b}{\left(1+q_{1}\right)^{2}}, \frac{\left(1+q_{2}+q_{2}^{2}\right) c+q_{2} d}{\left(1+q_{2}\right)^{2}}\right) \\
& +\Psi\left(\frac{\left(2 q_{1}+q_{1}^{2}\right) a+b}{\left(1+q_{1}\right)^{2}}, \frac{q_{2} c+\left(1+q_{2}+q_{2}^{2}\right) d}{\left(1+q_{2}\right)^{2}}\right) \\
\leq & \frac{1+q_{1}}{2(b-a)}\left[\int_{a}^{\frac{q_{1} a+b}{1+q_{1}}} \Psi\left(u, \frac{\left(1+q_{2}+q_{2}^{2}\right) c+q_{2} d}{\left(1+q_{2}\right)^{2}}\right){ }_{a} d_{q_{1}} u\right. \\
& +\int_{\frac{q_{1} b+a}{1+q_{1}}}^{b} \Psi\left(u, \frac{q_{2} c+\left(1+q_{2}+q_{2}^{2}\right) d}{\left(1+q_{2}\right)^{2}}\right)^{b} d_{q_{1}} u \\
& +\int_{\frac{q_{1} b+a}{1+q_{1}}}^{b} \Psi\left(u, \frac{\left(1+q_{2}+q_{2}^{2}\right) c+q_{2} d}{\left(1+q_{2}\right)^{2}}\right){ }^{b} d_{q_{1}} u \\
& \left.+\int_{a}^{\frac{q_{1} a+b}{1+q_{1}}} f\left(u, \frac{q_{2} c+\left(1+q_{2}+q_{2}^{2}\right) d}{2 q_{2}(d-c)}\right) \int_{a} d_{q_{1}} u\right] \\
& \left(1+q_{2}\right)^{2} \\
& +\frac{q_{2}}{1+q_{2}} \\
&
\end{aligned}
$$




$$
\begin{aligned}
& +\int_{c}^{\frac{q_{2} d+c}{1+q_{2}}} \Psi\left(\frac{a+\left(2 q_{1}+q_{1}^{2}\right) b}{\left(1+q_{1}\right)^{2}}, v\right){ }_{c} d_{q_{2}} v \\
& \left.+\int_{\frac{q_{2} c+d}{1+q_{2}}}^{d} \Psi\left(\frac{\left(2 q_{1}+q_{1}^{2}\right) a+b}{\left(1+q_{1}\right)^{2}}, v\right) d d_{q_{2}} v\right] \\
& \leq \frac{\left(1+q_{1}\right)\left(1+q_{2}\right)}{q_{2}(b-a)(d-c)}\left[\int_{a}^{\frac{q_{1} a+b}{1+q_{1}}} \int_{c}^{\frac{q_{2} d+c}{1+q_{2}}} \Psi(u, v)_{c} d_{q_{2}} v_{a} d_{q_{1}} u\right. \\
& +\int_{\frac{q_{1} b+a}{1+q_{1}}}^{b} \int_{\frac{q_{2} c+d}{1+q_{2}}}^{d} \Psi(u, v)^{d} d_{q_{2}} v^{b} d_{q_{1}} u \\
& \left.+\int_{\frac{q_{1} b+a}{1+q_{1}}}^{b} \int_{c}^{\frac{q_{2} d+c}{1+q_{2}}} \Psi(u, v){ }_{c} d_{q_{2}} v^{b} d_{q_{1}} u+\int_{a}^{\frac{q_{1} a+b}{1+q_{1}}} \int_{\frac{q_{2} c+d}{1+q_{2}}}^{d} \Psi(u, v)^{d} d_{q_{2}} v_{a} d_{q_{1}} u\right] \\
& \leq \frac{\left(1+q_{1}\right)}{2\left(1+q_{2}\right)(b-a)}\left[q_{2} \int_{a}^{\frac{q_{1} a+b}{1+q_{1}}} \Psi(u, c)_{a} d_{q_{1}} u+\int_{a}^{\frac{q_{1} a+b}{1+q_{1}}} \Psi\left(u, \frac{q_{2} d+c}{1+q_{2}}\right){ }_{a} d_{q_{1}} u\right. \\
& +\int_{\frac{q_{1} b+a}{1+q_{1}}}^{b} \Psi\left(u, \frac{q_{2} d+c}{1+q_{2}}\right){ }^{b} d_{q_{1}} u+q_{2} \int_{\frac{q_{1} b+a}{1+q_{1}}}^{b} \Psi(u, d)^{b} d_{q_{1}} u \\
& +q_{2} \int_{\frac{q_{1} b+a}{1+q_{1}}}^{b} \Psi(u, c)^{b} d_{q_{1}} u+\int_{\frac{q_{1} b+a}{1+q_{1}}}^{b} \Psi\left(u, \frac{q_{2} d+c}{1+q_{2}}\right){ }^{b} d_{q_{1}} u \\
& \left.+\int_{a}^{\frac{q_{1} a+b}{1+q_{1}}} \Psi\left(u, \frac{q_{2} c+d}{1+q_{2}}\right){ }_{a} d_{q_{1}} u+q_{2} \int_{a}^{\frac{q_{1} a+b}{1+q_{1}}} \Psi(u, d){ }_{a} d_{q_{1}} u\right] \\
& +\frac{\left(1+q_{2}\right)}{2 q_{2}\left(1+q_{1}\right)(d-c)}\left[q_{1} \int_{c}^{\frac{q_{2} d+c}{1+q_{2}}} \Psi(a, v){ }_{c} d_{q_{2}} v\right. \\
& +\int_{c}^{\frac{q_{2} d+c}{1+q_{2}}} \Psi\left(\frac{q_{1} a+b}{1+q_{1}}, v\right){ }_{c} d_{q_{2}} v \\
& +\int_{\frac{q_{2} c+d}{1+q_{2}}}^{d} \Psi\left(\frac{q_{1} b+a}{1+q_{1}}, v\right){ }^{d} d_{q_{2}} v+q_{1} \int_{\frac{q_{2} c+d}{1+q_{2}}}^{d} \Psi(b, v)^{d} d_{q_{2}} v \\
& +\int_{c}^{\frac{q_{2} d+c}{1+q_{2}}} \Psi\left(\frac{q_{1} b+a}{1+q_{1}}, v\right){ }_{c} d_{q_{2}} v+q_{1} \int_{c}^{\frac{q_{2} d+c}{1+q_{2}}} \Psi(b, v){ }_{c} d_{q_{2}} v \\
& \left.+q_{1} \int_{\frac{q_{2} c+d}{1+q_{2}}}^{d} \Psi(a, v)^{d} d_{q_{2}} v+\int_{\frac{q_{2} c+d}{1+q_{2}}}^{d} \Psi\left(\frac{q_{1} a+b}{1+q_{1}}, v\right) d^{d} d_{q_{2}} v\right] \\
& \leq \frac{q_{1} q_{2}[\Psi(a, c)+\Psi(a, d)+\Psi(b, c)+\Psi(b, d)]}{\left(1+q_{1}\right)\left(1+q_{2}\right)} \\
& +\frac{q_{1}\left[\Psi\left(b, \frac{q_{2} c+d}{1+q_{2}}\right)+\Psi\left(a, \frac{q_{2} d+c}{1+q_{2}}\right)+\Psi\left(a, \frac{q_{2} c+d}{1+q_{2}}\right)+\Psi\left(b, \frac{q_{2} d+c}{1+q_{2}}\right)\right]}{\left(1+q_{1}\right)\left(1+q_{2}\right)} \\
& +\frac{q_{2}\left[\Psi\left(\frac{q_{1} b+a}{1+q_{1}}, d\right)+\Psi\left(\frac{q_{1} b+a}{1+q_{1}}, c\right)+\Psi\left(\frac{q_{1} a+b}{1+q_{1}}, c\right)+\Psi\left(\frac{q_{1} a+b}{1+q_{1}}, d\right)\right]}{\left(1+q_{1}\right)\left(1+q_{2}\right)} \\
& +\frac{\Psi\left(\frac{q_{1} b+a}{1+q_{1}}, \frac{q_{2} c+d}{1+q_{2}}\right)+\Psi\left(\frac{q_{1} a+b}{1+q_{1}}, \frac{q_{2} c+d}{1+q_{2}}\right)+\Psi\left(\frac{q_{1} a+b}{1+q_{1}}, \frac{q_{2} d+c}{1+q_{2}}\right)+\Psi\left(\frac{q_{1} b+a}{1+q_{1}}, \frac{q_{2} d+c}{1+q_{2}}\right)}{\left(1+q_{1}\right)\left(1+q_{2}\right)} \\
& \leq \Psi(a, c)+\Psi(a, d)+\Psi(b, c)+\Psi(b, d) .
\end{aligned}
$$


Remark 2.5 If $q_{1}, q_{2} \rightarrow 1^{-}$, then Theorems $2.5-2.8$ give to the following inequalities, which is the refinement for the classical inequalities (1.1):

$$
\begin{aligned}
4 \Psi( & \left.\frac{a+b}{2}, \frac{c+d}{2}\right) \\
\leq & \Psi\left(\frac{3 a+b}{4}, \frac{3 c+d}{4}\right)+\Psi\left(\frac{3 a+b}{4}, \frac{3 d+c}{4}\right) \\
& +\Psi\left(\frac{a+3 b}{4}, \frac{3 c+d}{4}\right)+\Psi\left(\frac{a+3 b}{4}, \frac{c+3 d}{4}\right) \\
\leq & \frac{1}{b-a}\left[\int_{a}^{b} \Psi\left(u, \frac{3 c+d}{4}\right) d u+\int_{a}^{b} \Psi\left(u, \frac{c+3 d}{4}\right) d u\right] \\
& +\frac{1}{d-c}\left[\int_{c}^{d} \Psi\left(\frac{3 a+b}{4}, v\right) d v+\int_{c}^{d} \Psi\left(\frac{a+3 b}{4}, v\right) d v\right] \\
\leq & \frac{4}{(b-a)(d-c)} \int_{a}^{b} \int_{c}^{d} \Psi(u, v) d v d u \\
\leq & \frac{1}{2(b-a)}\left[\int_{a}^{b} \Psi(u, c) d u+2 \int_{a}^{b} \Psi\left(u, \frac{c+d}{2}\right) d u+\int_{a}^{b} \Psi(u, d) d u\right] \\
& +\frac{1}{2(d-c)}\left[\int_{c}^{d} \Psi(a, v) d v+2 \int_{c}^{d} \Psi\left(\frac{a+b}{2}, v\right) d v+\int_{c}^{d} \Psi(b, v) d v\right] \\
\leq & \frac{[\Psi(a, c)+\Psi(a, d)+\Psi(b, c)+\Psi(b, d)]}{4}+\frac{2 \Psi\left(a, \frac{c+d}{2}\right)+2 \Psi\left(b, \frac{c+d}{2}\right)}{4} \\
& +\frac{2 \Psi\left(\frac{a+b}{2}, c\right)+2 \Psi\left(\frac{a+b}{2}, d\right)}{4}+\frac{4 \Psi\left(\frac{a+b}{2}, \frac{c+d}{2}\right)}{4} \\
\leq & \Psi(a, c)+\Psi(a, d)+\Psi(b, c)+\Psi(b, d) .
\end{aligned}
$$

\section{Conclusion}

In this study, we have extended the definition of $q$-derivatives and $q$-integrals over the interval $[A, B]$ of the real lines. We have considered new $H-H$ inequalities in the context of $q$-calculus. For the desired results, we have developed an inequality with the same lower and upper estimates as in classical Theorem 1.1. Also, we have established new midpoint $H-H$ type inequalities, which confirm the refinements to the previously known inequalities.

Our results suggest that two different partitions exist for midpoint type inequalities in the $q$-analogues. Indeed, for the interval $[A, B]$, we have $A \leq \frac{q A+B}{1+q} \leq B$ and $A \leq \frac{A+q B}{1+q} \leq B$ with $q \in(0,1)$. Similarly for $[C, D]$, we have $C \leq \frac{q C+D}{1+q} \leq D$ and $C \leq \frac{C+q D}{1+q} \leq D$ with $q \in$ $(0,1)$. The last four inequalities use all partitions for the desired results. We believe that the results of this paper can be extended to establish new inequalities via different kinds of convex functions in the premises of $q$-calculus.

\section{Acknowledgements}

This work was supported by the Taif University Researchers Supporting Project (No. TURSP-2020/155), Taif University, Taif, Saudi Arabia, and the Deanship of Scientific Research at Princess Nourah Bint Abdulrahman University through the Fast-Track Research Funding Program. 
Availability of data and materials

No data were used to support this study.

\section{Competing interests}

The authors declare that they have no competing interests.

\section{Authors' contributions}

All authors contributed equally and significantly in writing this article. All authors read and approved the final manuscript.

\section{Author details}

'Department of Mathematical Sciences, Faculty of Sciences, Princess Nourah Bint Abdulrahman University, P.O. Box 84428, Riyadh 11671, Saudi Arabia. ${ }^{2}$ Department of Mathematics, Faculty of Technical Science, University Ismail Qemali, Vlora, Albania. ${ }^{3}$ Department of Mathematics, College of Education, University of Sulaimani, Sulaimani, Kurdistan Region, Iraq. ${ }^{4}$ Department of Mathematics and General Sciences, Prince Sultan University, P.O. Box 66833, Riyadh 11586, Saudi Arabia. ${ }^{5}$ Department of Medical Research, China Medical University, Taichung 40402, Taiwan. ${ }^{6}$ Department of Computer Science and Information Engineering, Asia University, Taichung, Taiwan. ${ }^{7}$ School of Natural Sciences, National University of Sciences and Technology, Islamabad, Pakistan. ${ }^{8}$ Department of Mathematics and Statistics, College of Science, Taif University, P. O. Box 11099, Taif 21944, Saudi Arabia.

\section{Publisher's Note}

Springer Nature remains neutral with regard to jurisdictional claims in published maps and institutional affiliations.

\section{Received: 5 January 2021 Accepted: 9 May 2021 Published online: 20 May 2021}

\section{References}

1. Niculescu, C., Persson, L.E.: Convex functions and their applications. Springer, New York (2006)

2. Peajcariaac, J.E., Tong, Y.L.: Convex functions, partial orderings, and statistical applications. Academic Press, San Diego (1992)

3. Beckenbach, E.F.: Convex functions. B. Am. math. soc. 1948(54), 439-460

4. Mohammed, P.O., Abdeljawad, T., Alqudah, M.A., Jarad, F.: New discrete inequalities of Hermite-Hadamard type for convex functions. Adv. Differ. Equ. 2021, 122 (2021)

5. Mohammed, P.O., Abdeljawad, T., Baleanu, D., et al.: New fractional inequalities of Hermite-Hadamard type involving the incomplete gamma functions. J. Inequal. Appl. 2020, 263 (2020)

6. Hadamard, J.: Étude sur les propriétés des fonctions entières en particulier d'une fonction considérée par Riemann. J. Math. Pures Appl. 1893(58), 171-215

7. Sarikaya, M.Z., Set, E., Yaldiz, H., Başak, N.: Hermite-Hadamard's inequalities for fractional integrals and related fractional inequalities. Math. Comput. Model. 2013(57), 2403-2407

8. Sarikaya, M.Z., Yildirim, H.: On Hermite-Hadamard type inequalities for Riemann-Liouville fractional integrals. Miskolc Math. Notes 2017(17), 1049-1059

9. Mohammed, P.O., Brevik, I.: A new version of the Hermite-Hadamard inequality for Riemann-Liouville fractional integrals. Symmetry 2020(12), 610

10. Baleanu, D., Mohammed, P.O., Zeng, S.: Inequalities of trapezoidal type involving generalized fractional integrals. Alex. Eng. J. 2020(59), 2975-2984

11. Mohammed, P.O.: Hermite-Hadamard inequalities for Riemann-Liouville fractional integrals of a convex function with respect to a monotone function. Math. Meth. Appl. Sci., 1-11 (2019). https://doi.org/10.1002/mma.5784

12. Mohammed, P.O., Sarikaya, M.Z., Baleanu, D.: On the generalized Hermite-Hadamard inequalities via the tempered fractional integrals. Symmetry 2020(12), 595

13. Mohammed, P.O., Aydi, H., Kashuri, A., Hamed, Y.S., Abualnaja, K.M.: Midpoint inequalities in fractional calculus defined using positive weighted symmetry function kernels. Symmetry 2021(13), 550

14. Dragomir, S.S.: On the Hadamard's inequality for convex functions on the co-ordinates in a rectangle from the plane. Taiwanese J. Math. 5(4), 775-788 (2001)

15. Mohammed, P.O.: Some new Hermite-Hadamard type inequalities for MT-convex functions on differentiable coordinates. J. King Saud Univ. Sci. 2018(30), 258-262

16. Baleanu, D., Kashuri, A., Mohammed, P.O., et al.: General Raina fractional integral inequalities on coordinates of convex functions. Adv. Differ. Equ. 2021, 82 (2021)

17. Özdemir, M.E., Yildiz, C., Akdemir, A.O.: On some new the Hadamard-type inequalities for co-ordinated quasi-convex functions. Hacet. J. Math. Stat. 2012(41), 697-707

18. Latif, M.A., Rashid, S., Dragomir, S.S., Chu, Y.M.: Hermite-Hadamard type inequalities for co-ordinated convex and quasi-convex functions and their applications. J. Inequal. Appl. 2019, 1 (2019)

19. Chen, F.X.: A note on the Hermite-Hadamard inequality for convex functions on the co-ordinates. J. Math. Inequal. 2014(8), 915-923

20. Jackson, F.H.: On q-functions and a certain difference operator. Trans. Roy. Soc. Edin. 1908(46), 253-281

21. Jackson, F.H.: On q-definite integrals. Quart. J. Pure Appl. Math. 1910(41), 193-203

22. Ernst, T:: A Comprehensive Treatment of q-Calculus. Springer, Basel (2012)

23. Gauchman, H.: Integral inequalities in q-calculus. Comput. Math. Appl. 47, 281-300 (2004)

24. Kac, V., Cheung, P.: Quantum calculus. Springer, Berlin (2001)

25. Tariboon, J., Ntouyas, S.K.: Quantum calculus on finite intervals and applications to impulsive difference equations. Adv. Diff. Equ. 2013, 282 (2013)

26. Tariboon, J., Ntouyas, S.K.: Quantum integral inequalities on finite intervals. J. Inequal. Appl. 2014, 121 (2014)

27. Alp, N., Sarikaya, M.Z., Kunt, M., İşcan, I.: q-Hermite-Hadamard inequalities and quantum estimates for midpoint type inequalities via convex and quasi-convex functions. J. King Saud Univ. 2018(30), 193-203 
28. Bermudo, S., Kórus, P., Nápoles Valdes, J.E.: On q-Hermite-Hadamard inequalities for general convex functions. Acta Math. Hungar. https://doi.org/10.1007/s10474-020-01025-6

29. Latif, M.A., Dragomir, S.S., Momoniat, E.: Some $q$-analogues of Hermite-Hadamard inequality of functions of two variables on finite rectangles in the plane. J. King Saud Univ. 2017(29), 263-273

30. Alp, N., Sarikaya, Quantum, M.Z.: Hermite-Hadamard's type inequalities for co-ordinated convex functions. Appl. Math. E-Notes 2020(20), 341-356

Submit your manuscript to a SpringerOpen ${ }^{\circ}$ journal and benefit from:

- Convenient online submission

$\checkmark$ Rigorous peer review

- Open access: articles freely available online

- High visibility within the field

- Retaining the copyright to your article

Submit your next manuscript at $\gg$ springeropen.com 